\title{
Maximal extension of conformally flat globally hyperbolic space-times
}

\author{
Clara Rossi Salvemini
}

May 7, 2018

\begin{abstract}
The notion of maximal extension of a globally hyperbolic space-time arises from the notion of maximal solutions of the Cauchy problem associated to the Einstein's equations of general relativity. Choquet-Bruhat and Geroch proved ([9]) that if the Cauchy problem has a local solution, this solution has a unique maximal extension. Since the causal structure of a space-time is invariant under conformal changes of metrics we may generalize this notion of maximality to the conformal setting. In this article we focus on conformally flat space-times of dimension greater or equal than 3. In this case, by a Lorentzian version of Liouville's theorem, these space-times are locally modeled on the Einstein space-time. In the first part we use this fact to prove the existence and uniqueness of the maximum extension for globally hyperbolic conformally flat space-times. In the second part we find a causal characterization of globally hyperbolic conformally flat maximal space-times whose developing map is a global diffeomorphism.
\end{abstract}

\section{Contents}

1 Introduction 1

2 Preliminaries 6

2.1 Causal structure of space-times . . . . . . . . . . 6

2.2 Conformally flat space-times . . . . . . . . . . . . 10

$3 C_{0}$-maximum extension

3.1 Cauchy-embeddings. . . . . . . . . . . . . . 15

3.2 Existence and uniqueness of the $C_{0}$-maximum extension . . 16

4 Complete $C_{0}$-maximal space-times 25

\section{Introduction}

Cauchy problem associated to the Einstein equation. The concept of maximal extension of a globally hyperbolic space-time comes 
from a PDE problem: the existence and uniqueness of maximal solutions for the Cauchy problem associated to the Einstein equation. The Einstein equation relates a physical object, the stress-energy tensor, with a geometric one, the curvature tensor of the universe. We can write it as following:

$$
\operatorname{Ric}(g)-1 / 2 \operatorname{scal}(g) g+\Lambda g=8 \pi T
$$

where $g$ is a Lorentzian metric, $\operatorname{Ric}(g)$ is the Ricci tensor, $\operatorname{scal}(g)$ is the scalar curvature, $\Lambda$ the cosmological constant and $T$ the stress-energy tensor which is a symmetric tensor of type $(2,0)$.

In vacuum the tensor $T$ is zero and the equation becomes: $\operatorname{Ric}(g)=$ 0 . A solution for this equation is just a Lorentzian manifold with Ricci curvature zero. In the general case, the meaning of what constitute a solution is not clear, because the topology of the universe and the stressenergy tensor are not defined a priori. A possible strategy to find solutions of the equation (1) is to assume that the solution is globally hyperbolic. By Geroch's Theorem ( 12 and 24, p. 1155]) every globally hyperbolic space-time is diffeomorphic to a product $S \times \mathbb{R}$, so that every slice $S \times\{t\}$ is a spacelike submanifold. Then we can formulate a Cauchy problem associated to the equation (1) as follows. The initial data is a Riemannian manifold $(S, h)$ of dimension $n$ equipped with a symmetric $(2,0)$-tensor $I I$, and a solution is a Lorentzian metric $g$ over the product manifold $M:=S \times \mathbb{R}$ such that $g$ verifies the equation (11) for a tensor $T$ given $a$ priori on $M$ and $I I$ is the shape tensor of the sub-manifold $S \times\{0\}$ of $M$. It turns out that a necessary condition to have a solution is that $h$ and $I I$ verify some equations, named the constraint equations of general relativity (14, ch.7 ) when $T=0$. Geroch and Choquet-Bruhat proved (in [8] ) that, when $T=0$, the constraint equations are also a sufficient condition to the existence and unicity of local solutions of the Cauchy problem. One may ask how the solutions develop far away from the initial data. Is it possible to have different developments out of a neighborhood of the initial data? We say that a solution $M$ extends another $N$, if $N$ is isometric to a neighborhood of the initial data in $M$. A maximal solution is then a solution which has only trivial extensions. In 9 Choquet-Bruhat et Geroch have proved:

Theorem 1.1. Any local solution $(M, g)$ of the Cauchy's problem has a maximal extension, which is unique up to isometry.

Maximal extension into a given category. It is well known that Theorem 1.1 naturally generalizes to larger families of space-times which are not necessarily solutions of the Einstein equations. Let start by a definition of maximality for globally hyperbolic space-times:

Definition 1.2. Let $M$ and $N$ be two globally hyperbolic space-times. An isometric embedding $f: M \rightarrow N$ is a Cauchy-embedding if there exists a Cauchy hypersurface $S \subset M$ such that $f(S)$ is a Cauchy hypersurface of $N$. In this case we say that $N$ extends $M$.

A globally hyperbolic space-time $M$ is maximal if every Cauchy-embedding of $M$ into an other space-time is onto. 
This more general notion of maximality coincides with the classical one in the case of space-times which are solutions of the same Cauchy problem. Therefore now the problem of existence and uniqueness of the maximal extension of a given globally hyperbolic space-time is well-defined even for space-times which are not solutions of the Cauchy problem.

The arguments involved in the proof of the existence of the maximal extension in Theorem 1.1 easily generalize: every globally hyperbolic spacetime admits a maximal extension, but in general there is no reason for this extension to be unique. However the maximal extension is unique if we consider "rigid categories" of space-times:

Definition 1.3. A category of space-times is a family $\mathscr{F}$ of space-times such that:

- $\mathscr{F}$ is stable by isometry: if $(M, g)$ is in $\mathscr{F}$ and $(N, h)$ is isometric to $(M, g)$ then $(N, h)$ is in $\mathscr{F}$.

- $\mathscr{F}$ is stable by restriction: if $(M, g)$ is in $\mathscr{F}$ then for every open set $U$ of $M,\left(U,\left.g\right|_{U}\right)$ is in $\mathscr{F}$.

- $\mathscr{F}$ is stable by gluing: if there is an open covering $\left(U_{i}\right)_{i \in I}$ of $(M, g)$ such that for every $i$ of $I$ the restriction $\left(U_{i},\left.g\right|_{U_{i}}\right)$ is in $\mathscr{F}$ then $(M, g)$ is in $\mathscr{F}$.

Definition 1.4. A space-time $M$ in a category $\mathscr{F}$ is $\mathscr{F}$-maximal if every Cauchy-embedding of $M$ into another space-time of the same category $\mathscr{F}$ is onto.

Again, the arguments for the existence in Theorem 1.1 apply: every spacetime in a category $\mathscr{F}$ always has a $\mathscr{F}$-maximal extension. The uniqueness comes from some additional hypothesis:

Definition 1.5. A category $\mathscr{C}$ of space-times is rigid if given two globally hyperbolic space-times $M$ and $N$ in $\mathscr{C}$, and an isometry $f: I^{ \pm}(p) \rightarrow$ $I^{ \pm}(q)$, where $p \in M$ and $q \in N$, then $f$ extends into an isometry $\hat{f}$ : $U \cup I^{ \pm}(p) \rightarrow V \cup I^{ \pm}(q)$, where $U$ and $V$ are neighborhoods of $p$ and $q$.

This is the key property used in the proof of the theorem of ChoquetBruhat and Geroch, who considered the category of space-times which are solutions of the same Cauchy problem; one of the steps of the proof is to show that this category is rigid.

Another important rigid category of space-times is the $(G, X)$-category, where $X$ is a fixed space-time and $G$ its isometry group. The elements of this category are space-times which are $(G, X)$-manifolds. It's easy to see that it is rigid: let $M$ and $N$ two such space-times and let $f$ : $I^{-}(p) \rightarrow I^{-}(q)$ an isometry such that $f(p)=q$ and let $\phi: U \rightarrow X$ and $\psi: V \rightarrow X$ two charts on the neighborhood of $p$ and $q$. By definition of $(G, X)$-manifolds, the isometry

$$
\psi \circ f \circ \psi^{-1}: \psi\left(V \cap I^{-}(q)\right) \rightarrow \psi\left(U \cap I^{-}(p)\right)
$$

extends into a unique element $g \in G$. Then the map $\hat{f}:=\psi \circ g \circ \phi$ is an isometry between $U$ and $V$.

Space-times of constant curvature are examples of $(G, X)$-manifolds: $X$ is 
the Minkowski space-time $\mathbb{R}^{1, n}$, when the curvature is zero, the de Sitter space-time $d \mathbb{S}_{1, n}$, when it is positive, and the anti-de Sitter space-time $A d \mathbb{S}_{1, n}$, when it is negative. Then we have notions of $\mathbb{R}^{1, n}$-maximal extension, $d \mathbb{S}_{1, n}$-maximal extension, and $A d \mathbb{S}_{1, n}$-maximal extension.

Since constant curvature space-times are solutions of the Einstein equation, by Theorem 1.1, these extensions are unique up to isometry. In fact, Theorem 1.1 is true for every rigid category:

Theorem 1.6. Every globally hyperbolic space-time in a rigid category $\mathscr{C}$ has a unique $\mathscr{C}$-maximal extension.

This statement is quite well-known by the experts of the field; in the present paper we will consider a slightly different problem, where we consider extensions by conformal embeddings, not necessarily isometric (see the next section). The tools and proof involved in this new context can be easily adapted to the isometric case, providing a complete proof of Theorem 1.6.

Maximal conformally flat extension. Since the causal structure is a conformal invariant, the notion of maximality defined in the previous section naturally generalizes to conformal classes of Lorentzian metrics. This is obtained by taking conformal Cauchy-embeddings, instead of isometric ones, in Definition 1.2. Then we say that a space-time $M$ is $C$ maximal if every conformal Cauchy-embedding of $M$ into another globally hyperbolic space-time is onto.

Even if here we are in the conformal context we can still use the language of category by just replacing the word "isometry" with "conformal diffeomorphism" in Definition 1.3. However, just as in the isometric case, the proof of the existence and uniqueness of the maximal extension requires some additional rigidity property. Moreover the family of conformally flat space-times is a sub-category of the $C$-category: we call it the $C_{0}$-category. So we can have a well-defined notion of $C_{0}$-maximality: a conformally flat space-time $M$ is $C_{0}$-maximal, if every conformal Cauchy-embedding of $M$ into another globally hyperbolic conformally flat space-time in onto.

Now that we have defined the notion of $C$-maximality and $C_{0}$-maximality, we can again ask the questions: does every conformally flat globally hyperbolic space-time have a $C$-maximal and $C_{0}$-maximal extension? Are these extensions unique up to conformal diffeomorphisms? The answer is the following generalization of Theorem 1.1 to the conformal and conformally flat case.

Theorem 1.7. Every globally hyperbolic conformally flat space-time $M$ of dimension $\geq 3$ has a unique $C_{0}$-maximal extension. This extension is unique up to conformal diffeomorphism.

In the section 3 of this article we give a proof of this result, using the fact that conformally flat space-times are $(G, X)$-manifolds, where $X$ is the Einstein space-time and $G$ its conformal group of diffeomorphisms. This gives an additional rigidity property: if we define the conformally rigid category by taking conformal diffeomorphisms, instead of isometries, in Definition 1.5 the $C_{0}$-category is conformally rigid.

A generalization of Theorem 1.7 to the $C$-category seems much more difficult to prove. We do not think that the techniques used here are enough 
to deal with the $C$-category. We do not know for the moment if a $C$ maximal extension does exist for every space-time, and if it is necessarily unique. The question is still open.

Summarizing, we have several notions of maximality for a globally hyperbolic space-time. These different notions are not completely independent but there are some implications. Just as in Riemannian geometry, constant curvature Lorentzian manifolds are conformally flat. Let $M$ be a constant curvature space-time. Then $M$ is a $(G, X)$-manifold (where $X$ is equal to $\mathbb{R}^{1, n}, A d S_{1, n}$ or $\left.d S_{1, n}\right)$, and we have:

$$
M \text { is } C \text {-maximal } \Rightarrow M \text { is } C_{0} \text {-maximal } \Rightarrow M \text { is } X \text {-maximal }
$$

The converse implications are not true in general: we could have a $C_{0}$-maximal space-time which is not $C$-maximal, or $X$-maximal spacetime which is not $C_{0}$-maximal, etc.. In another paper we will develop new tools which allow us to prove that in fact these two inverse implications are true: every $C_{0}$-maximal space-time and every $A d S_{1, n}$-maximal or $\mathbb{R}^{1, n}$ maximal space-time is also $C$-maximal. Conversely every $d S_{1, n}$-maximal space time always has a non trivial $C_{0}$-extension.

Completeness of $C_{0}$-maximal space-times. In the second part of the paper we study the developing map of a maximal conformally flat space-time. We provide a sufficient and necessary condition on the causal structure of the space-time for the developing map to be a global diffeomorphism; in other words, a causal characterization of conformally flat space-times which are complete as $(G, X)$-manifolds. The uniqueness of the $C_{0}$-maximal extension in Theorem 1.7 implies:

Theorem 1.8. The universal cover of the Einstein space-time is the only globally hyperbolic conformally flat space-time of dimension $\geq 3$ which is $C_{0}$-maximal, simply connected, and admitting a compact Cauchy hypersurface.

This result implies that a $C_{0}$-maximal globally hyperbolic conformally flat space-time $M$ is a finite quotient of the Einstein space-times if and only if the lift, to its universal cover, of every Cauchy hypersurface, is compact. Moreover, because we know very well the causal structure of the Einstein space-time, and in particular we have a very clear description of its lightlike geodesics, we have:

Theorem 1.9. Let $M$ be a conformally flat globally hyperbolic $C_{0}$-maximal space-time of dimension $\geq 3$ which has two freely homotopic lightlike geodesics which are distinct but with the same ends. Then $M$ is a finite quotient of universal cover of the Einstein space-time.

In a following paper we will show some consequences of this result. It gives some information about the domains of injectivity of the developing map of a $C_{0}$-maximal space-time. It turns out that the developing map of a conformally flat globally hyperbolic space-time $M$ has to be injective on the causal past and future of each point. Moreover, the image of these set is a regular Minkowski domain, future or past complete (following the definition of [6] and [1] to classify the $\mathbb{R}^{1, n}$-maximal globally hyperbolic space-time with compact Cauchy hypersurface). 
Organization of the paper. In Section 2 we recall some classical results in causality of space-time, in particular we recall the properties of globally hyperbolic space-times. We also give a detailed description of the Einstein space-time, which will be an essential tool in the proof of the mains results. The rigidity properties of conformal maps and Liouville's Theorem will be also recalled in this section. In section 3 after some properties of conformal Cauchy-embeddings, we give the proof of Theorem 1.7 In Section 4 we prove Theorem 1.8 and Theorem 1.9 .

\section{Preliminaries}

In this section we recall some classical definitions and results about conformally flat globally hyperbolic space-times and we gives the proof of some technical lemmas and propositions which play a rule in the proof of the main results of this article. The theory of space-times has been largely studied by Hawking, Penrose and many others. A quite complete exposition of the main results can be found in 14, 4, 20, 21. For a clear exposition of the hierarchy of causal notions associated to space-times see also [19].

\subsection{Causal structure of space-times}

Space-times. A Lorentzian $(n+1)$-manifold is a smooth $(n+1)$ manifold $M$ (which includes the topological assumption that $\mathrm{M}$ is metrizable and with countable basis), endowed with a symmetric non-degenerate 2-form $g$ with signature $(n, 1)$.

A non zerd 1 tangent vector $v$ is spacelike (resp. timelike, lightlike, causal) if $g(v, v)$ is positive (resp. negative, null, non-positive).

In each tangent space $T_{p} M$ the cone of causal vectors has two connected components $C_{p}^{+}$and $C_{p}^{-}$. The manifold $M$ is time-orientable if it is possible to make a continuous choice, in each tangent space of $M$, of one of them. This means that over $M$ there are two continuous cone fields: one of them is chosen to be the future one, denoted by $C^{+}$, and the other to be the past one, denoted by $C^{-}$. Such a choice defines a time-orientation on $M$. A non-spacelike vector $w \in T_{p} M$ is future-directed if it is in $C^{+}$ and past-directed if it is in $C^{-}$. Remark that up to a double cover $M$ is always time-orientable.

Definition 2.1. A space-time is a connected orientable and time-orientable Lorentzian manifold provided with a time-orientation.

A differentiable causal curve (respectively timelike, spacelike, lightlike) of a space-time $M$ is a $C^{1}$ map $\left.c:\right] a, b[\rightarrow M$ such that at every point its tangent vector is causal (respectively timelike, spacelike, lightlike). In particular, every causal curve $c$ is an immersion and the vectors $c^{\prime}(t)$ have the same time orientation for all $t \in] a, b[$ : the curve $c$ is said to be future or past oriented following the time-orientation of $c^{\prime}(t)$.

The causal future $J_{U}^{+}(A)$ (respectively chronological future $I_{U}^{+}(A)$ ) of a

\footnotetext{
${ }^{1}$ Our convention is to consider the zero vector as a spacelike vector. In particular, a causal or lightlike vector is non zero.
} 
subset $A$ of $M$ relative to an open set $U$ is the set of future ends of all future causal (respectively timelike) curves starting from a point of $A$ and contained in $U$. The chronological past and the causal past of $A$ relative to an open set $U$, noted $I_{U}^{-}(A)$ and $J_{U}^{-}(A)$, are the chronological and causal futures of $A$ for the opposite time-orientation on $M$. When $U=M$ the chronological and causal past an future of a set $A$ are noted $I^{ \pm}(A)$ and $J^{ \pm}(A)$. Since a timelike curve is in particular a causal curve we have $I_{U}^{ \pm}(A) \subset J_{U}^{ \pm}(A)$. These sets give what is called the causal structure of $M$. It's possible to give a more general notion of causal curve without changing the causal structure of $M$. A future causal curve is a $C^{0} \operatorname{map} c$ : ]$a, b\left[\subset \mathbb{R} \rightarrow M\right.$ such that for all $\left.t_{0} \in\right] a, b[$ and for all neighborhood $U$ of $c\left(t_{0}\right)$, there exists $\varepsilon>0$ such that for all $\left.t \in I:=\right] t_{0}-\varepsilon, t_{0}+\varepsilon$ [ we have $c(t) \in J_{U}^{-}\left(t_{0}\right)$ if $t \leq t_{0}$ and $c(t) \in J_{U}^{+}\left(t_{0}\right)$ if $t \geq t_{0}$. We can define the past causal curves like the future ones for the opposite chronological orientation.

It is not hard to prove that the causal curves just defined are more regular than continuous: they are locally Lipschitz (see 2] for the proof in Minkowski space-time). Moreover, by definition, it is clear that if there is a causal curve from a point $p$ to a point $q$, then there is a piecewise differentiable causal curve from $p$ to $q$.

The causal structure naturally defines two relations: given $x, y \in M$, we write $x<y$ iff $x \in I^{-}(y)$ and $x \leq y$ iff $x \in J^{-}(y)$. They are called the causal relations of $M$. By the definition it is clear that the relations $<$ and $\leq$ are transitive and that the relation $\leq$ is reflexive. If $\leq$ is also antisymmetric we say that $M$ is a causal space-time. This means that we cannot have causal closed curves in the space-time and in this case $\leq$ is a partial order on $M$. The causal relations are more than transitive. Given $x, y, z \in M$ then $x \leq y$ and $y<z$ imply $x<z$ (and $x<y$ and $y \leq z$ imply $x<z$ ). This is a consequence of the following proposition:

Proposition 2.2 (20] p. 294). In a Lorentz manifold if $\alpha$ is a causal curve from $p$ to $q$ that is not a null pregeodesic, then there is a timelike curve from $p$ to $q$ arbitrarily close to $\alpha$.

Globally hyperbolic space-times. In Riemannian geometry it is often useful to consider an open neighborhood which is geodesically convex: this is the image by the exponential map restricted to some open neighborhood of zero. In a pseudo-Riemannian manifold we also have the exponential map and we can make a similar construction. However from the point of view of the causal structure there is another notion of convexity: an open set $U$ of a space-time is causally convex if every causal curve between two of its points is contained in $U$.

A natural hypothesis, if we are looking for space-times which are interesting from a physical point of view, is to require that the space-time contains no closed causal loop (physically time-travel is not allowed). For example, this is the case for causal space-times. However often this is not enough to be physically useful; for instance a curve should not return arbitrary near to its starting point. This is precisely what happens in strongly causal space-times: a space-time is strongly causal if every point has a causally convex neighborhood. 
Definition 2.3. A space-time $M$ is globally hyperbolic if it is strongly causal and for every $x, y \in M$ such that $y \in J^{-}(x)$, the intersection $J^{+}(y) \cap J^{-}(x)$ is compact.

This is the classical definition of globally hyperbolicity, however Sanchez has recently proved, in [5, that the hypothesis " $M$ is strongly causal" can be replaced by the hypothesis " $M$ is causal".

One of the main properties of globally hyperbolic space-times is the following 2:

Lemma 2.4 ( 4 p. 78 (corollary 3.32) and 20] p. 405 (Proposition 8)). Let $M$ be a globally hyperbolic space-time. Let $\left\{p_{n}\right\}_{n \in \mathbb{N}}$ and $\left\{q_{n}\right\}_{n \in \mathbb{N}}$ be two sequences of points in $M$ such that $p_{n} \rightarrow p$ and $q_{n} \leq p_{n}$ and let $\gamma_{n}$ be, for every $n$, a past causal curve from $p_{n}$ to $q_{n}$. Then:

- if $\exists q \neq p$ such that $q_{n} \rightarrow q$ and $q \leq p$, then the $\gamma_{n}$ have a limit curve going from $p$ to $q$ and which is past and causal,

- if the sequence $\left\{q_{n}\right\}_{n \in \mathbb{N}}$ is unbounded, then there exists a past inextensible causal curve starting from $p$ which is a limit curve for $\gamma_{n}$.

An achronal (acausal) subset of a space-time is a subset which intersects every timelike (causal) curve in at most one point.

Definition 2.5. Let $A$ be an achronal subset of $M$. The future Cauchy development (resp. past) of $A$, written $\mathscr{D}^{+}(A)$ (resp. $\left.\mathscr{D}^{-}(A)\right)$, is the set of points $x$ of $M$ in the chronological future (resp. past) of $A$ such that every past (future) inextensible causal curve starting from $x$ intersects $A$. The Cauchy development of $A$ is the union

$$
\mathscr{D}(A):=A \cup \mathscr{D}^{+}(A) \cup \mathscr{D}^{-}(A) .
$$

Proposition 2.6 (20] p. 421). Let $A$ be an achronal subset of $M$. If $\operatorname{int}(\mathscr{D}(A))$ is not empty, then it is globally hyperbolic.

Definition 2.7. A locally achronal subset $A$ of $M$ is said to be edgeless if for every $x$ of $A$ there exists a neighborhood $U$ of $x$ such that:

- $U \cap A$ is achronal relative to $U$ : every timelike curve contained in $U$ intersects $U \cap A$ at most in one point,

- every causal curve contained in $U$, which starts from a point of $I_{U}^{-}(x)$ and ends in $I_{U}^{+}(x)$, must intersect $U \cap A$.

A subset $V$ of a space-time $M$ is a past set (reps. future set) if $I^{-}(V) \subset V$ (resp. $\left.I^{+}(V) \subset V\right)$.

Lemma 2.8 ([20] p. 414 Corollary 26 and 27). The (non empty) boundary of a past (future) set $P$ is a closed achronal and edgeless topological hypersurface.

In general every locally achronal edgeless subset of a space-time $M$ is an embedded topological hypersurface (see Lemma 1.2.28 of [2] for Minkowski space-time).

\footnotetext{
${ }^{2}$ In fact, as explained in [21], the definition of limit curve has been fitted in order to have this convergence property for globally hyperbolic space-times.
} 
Lemma 2.9. Let $A$ be an achronal edgeless subset of a strongly causal space-time $M$. Then if $\mathscr{D}^{+}(A)=\emptyset\left(\mathscr{D}^{-}(A)=\emptyset\right)$, for every point $p \in A$ there exists a past (future) inextensible lightlike geodesic c $:[0, \infty[\rightarrow M$ starting from $p$ such that $c \cap A$ is a past (future) lightlike geodesic contained in $A$ without past (future) limit point in $A$.

Proof. This lemma results from the theory of the Cauchy horizon for the Cauchy development of achronal subsets. A proof can be found in [14] p. 203 Proposition 6.5.3 and its corollary.

Definition 2.10. A Cauchy hypersurface of $M$ is a closed achronal edgeless set $S \subset M$ such that $\mathscr{D}(S)=M$.

$A$ Cauchy time-function is a continuous map $t: M \rightarrow \mathbb{R}$ such that, for every inextensible future causal curve $c$ of $M, t \circ c$ is increasing and onto. In particular every level set of $t$ is a Cauchy hypersurface for $M$.

By Proposition 2.6] if $M$ has a Cauchy hypersurface then it is globally hyperbolic. The converse is a consequence of the following more general result, called Geroch's Theorem.

Theorem 2.11. A space-time $M$ is globally hyperbolic if and only if it admits a $C^{\infty}$ Cauchy time-function.

This result, originally proved in [12, has been rewritten several times, in order to correct some mistakes in the proof of the regularity of the Cauchy time function. Another reference for the proof of the existence of continuous Cauchy time function is 24, p. 1155].

Corollary 2.12. Every globally hyperbolic space-time is homeomorphic to a product $S \times \mathbb{R}$. Furthemore, for every $t \in \mathbb{R}$, the projection on to the factor $\mathbb{R}$ is a Cauchy time-function.

In general it is not true that a closed achronal edgeless subset $A$ of a globally hyperbolic space-time is a Cauchy hypersurface 3 . However this is true under a compactness hypothesis:

Proposition 2.13. Let $S$ be an achronal edgeless compact subset of a globally hyperbolic space-time $M$. Then $S$ is a Cauchy hypersurface of $M$.

Proof. By hypothesis $S$ is closed achronal edgeless so we just have to verify that $S$ intersects every inextensible causal curve of $M$.

We start by proving that $\partial I^{-}(S)=\partial I^{+}(S)=S$. Assume by contradiction that there exists $p \in \partial I^{-}(S) \backslash S$. Let $\left\{p_{n}\right\}_{n \in \mathbb{N}}$ a sequence in $I^{-}(S)$ converging to $p$. For every $n$ there exists a point $z_{n}$ in $S$ such that $p_{n} \in$ $I^{-}\left(z_{n}\right)$. Since $S$ is compact, up to a subsequence, we can assume that the sequence $\left\{z_{n}\right\}_{n \in \mathbb{N}}$ converges to a point $z$. Then Lemma 2.4 applies: there exists a past causal curve $\gamma$ from $z$ to $p$. Since $p \in \partial I^{-}(S) \backslash S$; the curve $\gamma$ has to be a lightlike geodesic (according to Proposition 2.2). This implies that every point $p^{\prime} \neq p$ which is in $\gamma$ is also in $\partial I^{-}(S) \backslash S$.

Let $c$ be a timelike curve from a point $p^{\prime}$ in $\gamma$ to a point $z^{\prime} \in I^{+}(z)$. Since $S$ is edgeless, if $p^{\prime}$ and $z^{\prime}$ are sufficiently near $z$, the curve $c$ has to meet $S$

\footnotetext{
3 The set $A=\left\{x \in \mathbb{R}^{1, n}:\|x\|_{1, n}=-1\right\}$ is a closed achronal edgeless subset of $\mathbb{R}^{1, n}$. But it is not a Cauchy hypersurface, because no lightlike straight lines going through the origin intersect $A$.
} 
in a point $q$. By Proposition 2.2 we have $p \in I^{-}(q)$, and then $p \in I^{-}(S)$, which is a contradiction.

A similar argument can be used to prove $\partial I^{+}(S)=S$, so we have the disjoint union $M=I^{+}(S) \sqcup S \sqcup I^{-}(S)$.

Let $\tau: M \rightarrow \mathbb{R}$ be a Cauchy time function. Since $S$ is compact $\left.\tau\right|_{S}$ has a maximum $A$. We define $\Sigma:=\tau^{-1}(b)$ where $b>A$ : then $\Sigma$ is a Cauchy hypersurface contained in $I^{+}(S)$.

Let $p$ be a point in $I^{-}(S)$ and let $\alpha$ be a future inextensible causal curve starting from $p$. The curve $\alpha$ intersects $\Sigma$. Since $\Sigma$ is strictly contained in $I^{+}(S), \alpha$ must intersect the boundary of $I^{-}(S)$ before intersecting $\Sigma$. Since the boundary of $I^{+}(S)$ is $S, \alpha$ intersects $S$.

In the same way we can show that every past inextensible causal curve starting from of $I^{+}(S)$ must intersect $S$. This shows that $\mathcal{D}(S)=M$ and thus $S$ is a Cauchy hypersurface.

\subsection{Conformally flat space-times}

Conformal maps and causality. There is a natural question to ask: when do two different Lorentzian metrics define the same causal structure on a manifold? A sufficient condition is that the two metrics be in the same conformal class. Indeed, if in each tangent space we multiply the metric by a positive constant, the causal type of the tangent vectors does not change, and so the causal structure of the entire manifold is preserved. Then every result about the causal structure of a given space-time is true for all the Lorentzian metrics in the same conformal class. However, in general, it is clear that two different metrics in the same conformal class have different geodesics. This is because the Levi-Civita connexion is not preserved by conformal changes. The formula which gives the new LeviCivita connexion, after a conformal change of metric, can be found in [4], chapter 9. By this formula it is not hard to prove:

Lemma 2.14 (4], Proposition 9.17). Let $(M, g)$ a space-time, and $f$ : $(M, g) \rightarrow(M, g)$ a conformal map. Then the image by $f$ of every lightlike geodesic of $M$, up to parametrization, is a lightlike geodesic of $N$

A different and nice proof of Lemma 2.14, using the fact that lightlike geodesics are the solutions in the zero level of a Hamiltonian system, can be found in [10] (chapter 1, p. 14).

Einstein space-time. It is well known that the conformal sphere can be identified to the boundary of the hyperbolic space of higher dimension. This construction has a Lorentzian analog: the Einstein space-time can be identified to the conformal boundary of the anti-de Sitter space-time. Let $\mathbb{R}^{2, n+1}$ be the vector space $\mathbb{R}^{n+3}$ with the canonical quadratic form

\footnotetext{
${ }^{4}$ For intellectual satisfaction we should also mention that this condition is also necessary for strongly causal space-times. More precisely: in 15 S.W. Hawking, A.R. King and P.J.W. McCarthy have shown that if $g$ and $g^{\prime}$ define the same causal structure over $M$ and if this structure is strongly causal, then $g$ and $g^{\prime}$ are conformally equivalent. The hard part of their proof is to show that every homeomorphism which preserves the causal structure of a strongly causal space-time is differentiable. Starting from that it is easy to show that the identity is a conformal map
} 
of signature $(2, n+1)$ and let $C$ be the cone of isotropic vectors. Let $S\left(\mathbb{R}^{2, n+1}\right)$ the quotient of $\mathbb{R}^{2, n+1}$ by positive rescaling, and let $\pi: \mathbb{R}^{2, n+1} \rightarrow$ $S\left(\mathbb{R}^{2, n+1}\right)$ be the associated projection. Since the space-time $A d \mathbb{S}_{1, n}$ is defined as the set of vectors of $\mathbb{R}^{2, n+1}$ which have norm -1 , the map $\pi$ is injective on this set. The space-time $A d \mathbb{S}_{1, n}$ is then identified to its image, and we call it the projective model of $A d \mathbb{S}_{1, n}$. The boundary of the image of $A d \mathbb{S}_{1, n}$ by $\pi$ is the image of $C$.

For every $v \in \mathbb{R}^{2, n+1}$ the kernel of $d_{v}\left(\left.\pi\right|_{C}\right)$ is exactly the degenerate direction of the ambient quadratic form of $\mathbb{R}^{2, n+1}$ restricted to $T_{p} C$. Given two sections $\phi, \phi^{\prime}: \pi(C) \rightarrow C$, there is always a positive function $f$ defined on $\pi(C)$ such that for every $x \in \pi(C)$, we have $\phi(x)=f(x) \phi^{\prime}(x)$. The two metrics defined on $\pi(C)$ by the pull backs by $\phi$ and $\phi^{\prime}$ of the quadratic form of $\mathbb{R}^{2, n+1}$, are then conformally equivalent, with conformal factor $f^{2}$. In other words, the quadratic form of $\mathbb{R}^{2, n+1}$ naturally defines a conformal class of Lorentzian metrics $[g]$ over $\pi(C)$.

Definition 2.15. The Einstein space-time of dimension $n+1$, denoted Ein $_{1, n}$, is the topological space $\pi(C)$ endowed with the conformal class of Lorentzian metrics $[\mathrm{g}]$.

It turns out that the conformal class $[g]$ of $\operatorname{Ein}_{1, n}$ is conformally flat: that is, every point $p$ of $\operatorname{Ein}_{1, n}$ has a neighborhood $U$ such that $\left.[g]\right|_{U}$ contains a flat metric. This fact is not evident a priori, it comes from the fact that the model flat space-time, the Minkowski space-time $\mathbb{R}^{1, n}$, admit conformal embeddings in $\operatorname{Ein}_{1, n}$. Since the action of the group $O(2, n+1)$ on $\operatorname{Ein}_{1, n}$ is transitive, every point of the space-times $\operatorname{Ein}_{1, n}$, has a neighborhood conformally equivalent to $\mathbb{R}^{1, n}$.

Moreover, the other two models of constant curvature space-times, $d \mathbb{S}_{1, n}$ and $A d \mathbb{S}_{1, n}$, also conformally embeds in $\operatorname{Ein}_{1, n}$, then we obtain that every constant curvature space-times is conformally flat (see [3] Proposition 2.3 for the $A d \mathbb{S}$ case, see 22 and section 2.3 of 11 for the others). This situation is similar to the Riemannian case: the euclidean and the hyperbolic space conformally embed into the Riemannian sphere, where the orthogonal group acts transitively.

Is not hard to see that the space-time $\operatorname{Ein}_{1, n}$ can be identified with the product $\mathbb{S}^{n} \times \mathbb{S}^{1}$, equipped with the conformal class of the metric $d \sigma^{2}-d \theta^{2}$, where $d \sigma^{2}$ and $d \theta^{2}$ are the canonical metrics over $\mathbb{S}^{n}$ and $\mathbb{S}^{1}$. This can be seen by looking at the intersection between $C$ and the sphere of radius 2 for the canonical euclidean metric of $\mathbb{R}^{n+3}$. By this identification $\operatorname{Ein}_{1, n}$ is clearly an orientable and time orientable manifold and thus a space-time. The orthogonal group $O(2, n+1)$ acts transitively and faithfully on $C$ and this action preserves straight lines. Hence $O(2, n+1)$ acts transitively and faithfully over $\operatorname{Ein}_{1, n}$ and preserves its conformal class of metrics.

Lemma 2.16. Every causal (timelike) curve $c$ of Ein E $_{1, n}$ can be parameterized as $\left(x(t), e^{i 2 \pi t}\right)$, where $x(t)$ is a (strictly) 1-Lipschitz map from an interval of $\mathbb{R}$ into $\mathbb{S}^{n}$. The lightlike geodesics of Ein $n_{1, n}$ are the causal curves $c$ such that, in the previous parametrization, $x(t)$ is a geodesic of $\mathbb{S}^{n}$ parameterized by its arc length.

Proof. Let $s \in I \subset \mathbb{R} \longmapsto c(s)=(w(s), p(s)) \in \mathbb{S}^{n} \times \mathbb{S}^{1}$ a future causal 
curve in $\operatorname{Ein}_{1, n}$. First suppose that $c$ is $C^{1}$ piecewise. Then $c$ must verify:

$$
\left\|w^{\prime}(s)\right\|^{2} \leq\left|p^{\prime}(s)\right|^{2}
$$

This implies that the vector $p^{\prime}(s)$, tangent to $\mathbb{S}^{1}$, never vanishes. The application $s \longmapsto p(s)$ can then be written as $p(s)=e^{i \phi(s)}$, where $s \longmapsto$ $\phi(s)$ is a monotone map from $I$ to an interval $J$ of $\mathbb{R}$. Since $c$ is a future causal curve the map $\phi(s)$ is a strictly increasing map. Then, changing the parameter $s$ into a parameter $t:=\phi(s)$, we have $c(t)=\left(x(t), e^{i t}\right)$, for all $t$ in $J$, where $x:=w \circ \phi^{-1}$, and $\left\|x^{\prime}(t)\right\|^{2} \leq 1$. If we integrate the formula between two points $t$ and $t^{\prime}$ of $J$, we have:

$$
d_{0}\left(x(t), x\left(t^{\prime}\right)\right) \leq\left|t-t^{\prime}\right|
$$

where $d_{0}$ is the distance over $\mathbb{S}^{n}$ for the canonical metric. The map $t \in$ $I \longmapsto x(t)$ is 1-Lipschitz; moreover $c$ is timelike if and only if the inequality (3) is strict, and (3) is strict if and only if the inequality in (4) is strict. Therefore, $C^{1}$ timelike curves are strictly 1-Lipschitz. The curve $c$ is a lightlike geodesic if and only if the inequality (3) is an equality, and this is true if and only if (3) is an equality, that is, if $x$ is a geodesic of $\mathbb{S}^{n}$. Hence, the lemma is proved for $C^{1}$ curves.

Now assume that $s \in I \longmapsto c(s)=(w(s), p(s))$ is a topological causal curve (not necessary $C^{1}$ ). By definition, given $s<s^{\prime}$ close one to the other, there exists a non trivial $C^{1}$ causal curve between $c(s)$ and $c\left(s^{\prime}\right)$. Since the result is proved in the case of $C^{1}$ curves, we have:

$$
0<d_{0}\left(w(s), w\left(s^{\prime}\right)\right) \leq\left|p(s)-p\left(s^{\prime}\right)\right|
$$

Therefore we can write $p(s)=e^{i \psi(s)}$, where $\psi(s)$ is strictly increasing map from $\mathbb{R}$ to an interval $K$ of $\mathbb{R}$, and then $c(t)=\left(x(t), e^{i t}\right)$, where $x:=w \circ \psi^{-1}$ satisfies the inequality (4), that is, $x$ is 1-Lipschitz. As before, we can see that $c$ is timelike if and only if $x$ is strictly 1-Lipschitz, and that $c$ is a lightlike geodesic if and only if $x$ is a geodesic of $\mathbb{S}^{n}$. The lemma is proved.

Corollary 2.17. Ein $_{1, n}$ is totally vicious, i.e. the past and the future of every point is the entire space-time.

Since $\operatorname{Ein}_{1, n}$ is totally vicious, its causal structure gives no information: every point is causally related to any other point. However its universal covering is globally hyperbolic and has a well understood causal structure.

The universal covering of the Einstein space-time, $\widetilde{\operatorname{Ein}_{1, n}}$, is identified to $\left(\mathbb{S}^{n} \times \mathbb{R},\left[d \sigma^{2}-d t^{2}\right]\right)$, where $d t^{2}$ is the canonical metric over $\mathbb{R}$. Let pr $: \widetilde{\operatorname{Ein}}_{1, n} \rightarrow \operatorname{Ein}_{1, n}$ be the covering map. The fundamental group of $\operatorname{Ein}_{1, n}$ is isomorphic to $\mathbb{Z}$ : it can be identified with the cyclic group generated by the map $\delta: \widetilde{\operatorname{Ein}}_{1, n} \rightarrow \widetilde{\operatorname{Ein}}_{1, n}$ which associates to $(x, t)$ the point $(x, t+2 \pi)$. This is clearly a conformal diffeomorphism of $\widetilde{\operatorname{Ein}}_{1, n}$.

The antipodal map of $\mathbb{R}^{2, n+1}, x \in \mathbb{R}^{2, n+1} \longmapsto-x$, defines a map $\bar{\sigma}$ : $\operatorname{Ein}_{1, n} \rightarrow \operatorname{Ein}_{1, n}$, which is the product of the two antipodal maps of $\mathbb{S}^{n}$ and $\mathbb{S}^{1}$. The map $\bar{\sigma}$ lifts to $\widetilde{\operatorname{Ein}}_{1, n}$ giving the map $\sigma: \widetilde{\operatorname{Ein}}_{1, n} \rightarrow \widetilde{\operatorname{Ein}}_{1, n}$ which associates to $(x, t) \in \mathbb{S}^{n} \times \mathbb{R}$ the point $(-x, t+\pi)$. Then $\sigma^{2}=\delta$. 


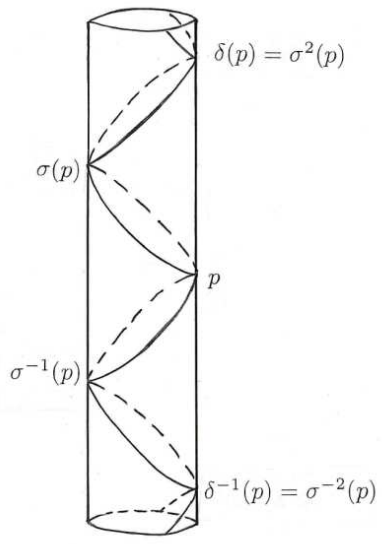

Figure 1: Two inextensibles lightlike geodesics in $\widetilde{\operatorname{Ein}_{1, n}} \simeq \mathbb{R} \times \mathbb{S}^{n}$

Definition 2.18. Two points $x$ and $y$ of $\widetilde{\operatorname{Ein}_{1, n}}$ are conjugate if one is the image by $\sigma$ of the other.

Since the projection $p r: \widetilde{\operatorname{Ein}}_{1, n} \rightarrow \operatorname{Ein}_{1, n}$ is a conformal map, given a causal curve $c:$ in $\widetilde{E i n}_{1, n}$, the curve $p r \circ c$ is a causal curve of $\operatorname{Ein}_{1, n}$. Then by Lemma 2.16 we have:

Lemma 2.19. Every causal curve $c$ of $\widetilde{\text { Ein }}_{1, n}$ can be parameterized as $c(t)=(x(t), t)$, where $x(t)$ is a 1-Lipschitz map from an interval of $\mathbb{R}$ into $\mathbb{S}^{n}$. The lightlike geodesics of $\widetilde{\text { Ein }_{1, n}}$ are the causal curves $c$ such that, for the previous parametrization, $x(t)$ is a geodesic of $\mathbb{S}^{n}$ parameterized by its arc length (see Figure 1).

A causal curve of $\widetilde{\operatorname{Ein}}_{1, n}$ is inextensible if the parametrization given by the previous lemma is defined for every $t$ in $\mathbb{R}$. It is then easy to see that $\widetilde{\operatorname{Ein}}_{1, n}$ is a globally hyperbolic space-time, with Cauchy hypersurfaces homeomorphic to $\mathbb{S}^{n}$ : the map $(x, t) \in \mathbb{S}^{n} \times \mathbb{R} \simeq \widetilde{\operatorname{Ein}}_{1, n} \rightarrow \mathbb{R}$ is a Cauchy time function.

Thanks to Lemma 2.19 we can understand the causal structure of $\widetilde{\operatorname{Ein}}_{1, n}$ :

Lemma 2.20. Let $p=(x, t) \in \widetilde{\operatorname{Ein}_{1, n}}$. Then

$$
\begin{aligned}
I^{ \pm}(p) & =\left\{\left(x^{\prime}, t^{\prime}\right) \in \widetilde{\operatorname{Ein}}_{1, n} / d_{0}\left(x, x^{\prime}\right)< \pm\left(t^{\prime}-t\right)\right\} \\
J^{ \pm}(p) & =\left\{\left(x^{\prime}, t^{\prime}\right) \in \widetilde{\operatorname{Ein}}_{1, n} / d_{0}\left(x, x^{\prime}\right) \leq \pm\left(t^{\prime}-t\right)\right\}
\end{aligned}
$$

where $d_{0}$ is the canonical distance on $\mathbb{S}^{n}$.

Remark 2.21. The inextensible lightlike geodesics starting from a point $p$ of $\widetilde{\operatorname{Ein}}_{1, n}$ have common intersections at all the points $\sigma^{k}(p)$, for $k \in \mathbb{Z}$. Outside these points, they are pairwise disjoint. 
Rigidity of conformal maps. Liouville's Theorem is originally a theorem of conformal Riemannian geometry stating that, in dimension $n \geq 3$, every conformal map between two open sets of the sphere is the restriction of an unique element of $O^{+}(1, n)$. This implies that the group of conformal transformations of the sphere $\mathbb{S}^{n}$ (also called the group of Möbius transformations) is exactly $O^{+}(1, n)$.

This theorem has been generalized by C. Frances (see [10] and [7]) to pseudo-Riemannian conformally flat metrics. This has been possible because the Liouville's Theorem is an aspect of a more general phenomena: the rigidity of conformal maps, between pseudo-Riemannian manifolds of dimension greater or equal than 3 .

Let $M$ and $N$ be two manifolds. Denote by $\operatorname{Diff}_{l o c}(M, N)$ the set of local diffeomorphism between $M$ and $N$. On $\operatorname{Diff}_{l o c}(M, N)$ we have the following equivalence relation: two local diffeomorphisms $f, g: M \rightarrow N$ are said to be equivalent if in some local chart they have the same Taylor polynomial up to order $r$ at $x$. The $r$-jet of $f \in \operatorname{Diff}_{l o c}(M, N)$ at a point $x \in M$, denoted by $j_{x}^{r} f$, is the equivalence class of $f$ for this relation. We have the following rigidity result:

Theorem 2.22. Let $M$ and $N$ be two pseudo-Riemannian manifolds of dimension $\geq 3$. Let $f$ and $g$ be two conformal maps from $M$ to $N$. If $f$ and $g$ have the same 2-jet at one point of $M$, then they are equal.

A proof of this result can be found in 10 (chapter 2). For more details about rigidity of conformal application see also [16] and 23 .

Liouville's Theorem is then a consequence of Theorem 2.22

Theorem 2.23. (Liouville) For every $n \geq 2$, every conformal map between two open sets of Ein $_{1, n}$ is the restriction of a unique element of $S O(2, n+1)$.

In particular this implies that the conformal group of $\operatorname{Ein}_{1, n}$ is exactly $O(2, n+1)$ and the group of conformal maps which preserve the orientation and the time-orientation is the connected component of the identity, that we denote by $O_{0}(2, n+1)$.

Every conformal diffeomorphism of $\operatorname{Ein}_{1, n}$ lifts to a conformal diffeomorphism of $\widetilde{\operatorname{Ein}}_{1, n}$. By Liouville's Theorem, when $n \geq 2$, the reverse statement is also true: every conformal diffeomorphism of $\widetilde{\operatorname{Ein}}_{1, n}$ defines a unique conformal diffeomorphism of $\operatorname{Ein}_{1, n}$. So we have a surjective morphism $j: \operatorname{Conf}\left(\widetilde{\operatorname{Ein}}_{1, n}\right) \rightarrow \operatorname{Conf}\left(\operatorname{Ein}_{1, n}\right)$. The kernel of $j$ is the subgroup generated by $\delta$; it is contained in the center of $\operatorname{Conf}\left(\widetilde{\operatorname{Ein}}_{1, n}\right)$. Then $\operatorname{Conf}\left(\widetilde{\operatorname{Ein}}_{1, n}\right)=O(\widetilde{2, n+1})=O(2, n+1) \rtimes \mathbb{Z}$.

Corollary 2.24. Every conformally flat space-time of dimension greater then 3 is locally modeled on $\left(\right.$ Ein $\left._{1, n+1}, O_{0}(2, n+1)\right)$.

The proof of this result is quite standard, it can be found for example in [17] in the case of Riemannian conformal geometry. In fact in Riemannian geometry we have the same situation: Liouville's Theorem implies that every conformally flat Remannnian manifold is locally modeled on the conformal sphere endowed with the action of its group of conformal transformations (see [17]). More details on the general theory of $(X, G)$ manifolds can be found in 13 . 


\section{$3 \quad C_{0}$-maximum extension}

\subsection{Cauchy-embeddings.}

In this section $M$ and $N$ will always assumed to be globally hyperbolic space-times with Cauchy hypersurfaces $S$ and $S^{\prime}$ respectively. We say that a conformal map $f: M \rightarrow N$ is a (conformal) Cauchy-embedding if $S^{\prime}=f(S) ; f$ is also denoted by $f:(M, S) \rightarrow\left(N, S^{\prime}\right)$. If moreover $f$ is an isometry, we say that $f$ is an isometric Cauchy-embedding.

To prove the existence and uniqueness of the conformally flat maximal extension we need some technical results about conformal Cauchy-embeddings. Since these results only involve causal properties, it makes no difference whether we consider conformal or isometric Cauchy-embeddings.

Lemma 3.1. The image of any conformal Cauchy-embedding $f:(M, S) \rightarrow$ $\left(N, S^{\prime}\right)$ is a causally convex open subset of $N$.

Proof. Let $x$ and $y$ be two points of $M$ such that there exists a future causal curve $\alpha:[0,1] \rightarrow N$ with $c(0)=f(x)$ and $c(1)=f(y)$. Let $c: \mathbb{R} \rightarrow N$ be an inextensible future causal curve which extends $\alpha$. Since $N$ is globally hyperbolic, $c: \mathbb{R} \rightarrow N$ is an embedding. Then, since $f(M)$ is an open subset of $N$, the intersection $c^{-1}(f(M) \cap c(\mathbb{R}))$ is the union of disjoint segments (the connected components). Let $I$ be one of them. The curve $C:=\left(\left.f\right|_{f(M)}\right)^{-1} \circ c: I \rightarrow M$ is a causal inextensible curve in $M$; therefore, $C(t)$ intersects the Cauchy hypersurface $S$ of $M$ at a point $z \in M$. It implies $f(z) \in f(S) \cap c(I) \neq \emptyset$. Since $f(S)$ is a Cauchy hypersurface it meets every causal curve at most at one point. Then $c^{-1}(f(M) \cap c(\mathbb{R}))$ has only one connected component which is the entire interval $[0,1]$. It follows that $f(M)$ is causally convex.

Corollary 3.2. The image of every achronal set $A$ of $M$ by any conformal Cauchy-embedding $f:(M, S) \rightarrow\left(N, S^{\prime}\right)$ is an achronal subset of $N$.

Proof. Assume by contradiction that there is a timelike curve $\gamma$ between two points of $f(A)$. Since $f(M)$ is causally convex in $N$, the curve $\gamma$ is completely contained in $f(M)$. Then the curve $\left(\left.f\right|_{f(M)}\right)^{-1} \circ \gamma$ is a timelike curve between two points of $A$ : this contradicts the hypothesis.

Corollary 3.3. A conformal Cauchy-embedding $f:(M, S) \rightarrow\left(N, S^{\prime}\right)$ sends every Cauchy hypersurface of $M$ to a Cauchy hypersurface of $N$.

Proof. Let $\Sigma \subset M$ a Cauchy hypersurface. According to Corollary 3.2 , $f(\Sigma)$ is an achronal hypersurface. Since $f(M)$ is an open neighborhood of $f(\Sigma)$ and $\Sigma$ is edgeless, $f(\Sigma)$ is edgeless too. We have to show that $f(\Sigma)$ intersects every inextensible causal curve. Let $c: \mathbb{R} \rightarrow N$ be an inextensible causal curve of $N$. We know that $c$ intersects $S^{\prime}$, and this implies $f(M) \cap c(\mathbb{R}) \neq \emptyset$. By the previous proof $\left(\left.f\right|_{f(M)}\right)^{-1} \circ c: \mathbb{R} \rightarrow M$ is a inextensible causal curve of $M$. Since $\Sigma$ is a Cauchy hypersurface it intersects this curve. Hence $c$ intersects $f(\Sigma)$ : it shows that $f(\Sigma)$ is a Cauchy hypersurface of $M^{\prime}$. 
Lemma 3.4. Let $U$ be an open neighborhood of the Cauchy hypersurface $S$ in $M$. Let $f, g: M \rightarrow N$ be two Cauchy-embeddings such that $\left.f\right|_{U}=\left.g\right|_{U}$. Then $f=g$.

Proof. The set $\mathcal{U}:=\left\{x \in M: j_{x}^{2} f=j_{x}^{2} g\right\}$ is a closed subset of $M$, which is non-empty since $f$ and $g$ coincide in $U$. By Theorem $2.22, \mathcal{U}$ is also open. Since $M$ is connected, we have $\mathcal{U}=M$.

Lemma 3.5. Let $f: M \rightarrow N$ be a Cauchy-embedding. The boundary $\partial f(M)$ is the union of two disjoint closed achronal edgeless subsets (each possibly empty) $\partial^{+} f(M)$ and $\partial^{-} f(M)$ of $N$ such that

$$
I^{-}\left(\partial^{+} f(M)\right) \cap I^{+}\left(\partial^{-} f(M)\right) \subset f(M) .
$$

Proof. Let $S$ be a Cauchy spacelike hypersurface of $M$. We identify $M$ with its image in $N$ by $f$; in particular, we consider $S$ as a Cauchy hypersurface of $N$. Let $N^{ \pm}:=I^{ \pm}(S) \cap N$ (where $I^{ \pm}(S)$ denote the future/past of $S$ in $N$ ) and let $M^{ \pm}:=N^{ \pm} \cap M$. The boundary $\partial M$ is then the disjoint union of $\partial^{+} M:=\partial M \cap N^{+}$and $\partial^{-} M:=\partial M \cap N^{-}$.

1) For every point $p \in \partial^{+} M$ we have $I^{-}(p) \cap N^{+} \subset M^{+}$.

Let $q \in I^{-}(p) \cap N^{+}$. There exists a past causal curve $c$ between $q$ and a point $z \in S$. For every $w$ sufficiently close to $p$ we have $q \in I^{-}(w)$. Since $p$ lies in the boundary of $M^{+}$, we can select such a $w$ in $M^{+}$. Then, there exists a past causal curve going from $w$ to $z$ through $q$. Since $M$ is causally convex in $N$ (Lemma 3.1) we obtain $q \in M^{+}$.

Reversing the time orientation we have also proved:

$\left.1^{\prime}\right)$ For every point $p \in \partial^{-} M: I^{+}(p) \cap N^{-} \subset M^{-}$. In particular,

$$
I^{-}\left(\partial^{+} f(M)\right) \cap I^{+}\left(\partial^{-} f(M)\right) \subset f(M) .
$$

2) $I^{+}\left(\partial^{+} M\right) \cap M$ is empty.

Assume by contradiction that there is an element $x$ of $I^{+}\left(\partial^{+} M\right) \cap M$. There exists a past causal curve $c$ between $x$ and a point $y$ in $\partial^{+} M$. Extend $c$ to an inextensible (in $N$ ) past causal curve $c^{\prime}$. Then $c^{\prime}$ intersects $S$ at a point $z$. Since $M$ is causally convex in $N$, consequently $y \in M$, contradicting the hypothesis.

3) $\partial^{+} M$ is achronal.

Let $c$ be a timelike future curve linking two points $x<y$ of $\partial^{+} M$. Then $I^{+}(x)$ is an open neighborhood of $y$, and by 2) it is disjoint from $M$ : but this contradicts the fact that $y$ lies in the closure of $M$.

4) $\partial^{+} M$ is edgeless.

Every causal curve between a point in $N^{+} \backslash M^{+}$and a point in $M^{+}$ intersects $\partial^{+} M$. By 2) we have that $\partial^{+} M$ is edgeless.

Reversing the time orientation we have the same results for $\partial^{-} M$.

\subsection{Existence and uniqueness of the $C_{0}$-maximum extension}

Let $M$ be a globally hyperbolic space-time of dimension $n+1 \geq 3$, and let $\phi: \Sigma \rightarrow M$ be a conformal embedding of a Riemannian manifold $\Sigma$ in $M$ such that $\phi(\Sigma)$ is a Cauchy hypersurface of $M$. Let $\mathcal{F}$ be the set of triples $(N, \psi, f)$, where: 
- $N$ is a globally hyperbolic space-time,

- $\psi: \Sigma \rightarrow N$ is a conformal embedding such that $\psi(\Sigma)$ is a Cauchy hypersurface of $N$,

- $f: M \rightarrow N$ is a conformal Cauchy-embedding such that $f \circ \phi=\psi$.

We can define the following relation over $\mathcal{F}$ :

$$
\begin{gathered}
(N, \psi, f) \preceq\left(N^{\prime}, \psi^{\prime}, f^{\prime}\right) \Longleftrightarrow \quad \exists h: N \rightarrow N^{\prime} \text { conformal embedding } \\
\text { such that } h \circ \psi=\psi^{\prime} .
\end{gathered}
$$

The fact that $h \circ \psi=\psi^{\prime}$ implies $h \circ f=f^{\prime}$. Moreover, by Lemma 3.4 if $(N, \psi, f) \preceq\left(N^{\prime}, \psi^{\prime}, f^{\prime}\right)$, then the Cauchy-embedding $h: N \rightarrow N^{\prime}$ such that $h \circ \psi=\psi^{\prime}$ is unique.

The relation $\preceq$ is clearly reflexive and transitive, but not antisymmetric. Nevertheless, if $(N, \psi, f) \preceq\left(N^{\prime}, \psi^{\prime}, f^{\prime}\right)$ and $\left(N^{\prime}, \psi^{\prime}, f^{\prime}\right) \preceq(N, \psi, f)$ then there are two Cauchy-embeddings $h: N \rightarrow N^{\prime}$ and $h^{\prime}: N^{\prime} \rightarrow N$ such that $h \circ \psi=\psi^{\prime}$ and $h^{\prime} \circ \psi^{\prime}=\psi$. The restriction of $h \circ h^{\prime}$ to the Cauchy hypersurface $\psi(\Sigma)$ is the identity, hence, by Lemma 3.4, $h \circ h^{\prime}$ is the identity map on $N$. Similarly, $h^{\prime} \circ h$ is the identity map of $N^{\prime}$. We have proved that $N$ and $N^{\prime}$ are conformally diffeomorphic.

In order to obtain a partial ordered set we consider over $\mathcal{F}$ the relation:

$$
\begin{aligned}
(N, \psi, f) \simeq\left(N^{\prime}, \psi^{\prime}, f^{\prime}\right) \Longleftrightarrow & (N, \psi, f) \preceq\left(N^{\prime}, \psi^{\prime}, f^{\prime}\right) \text { and } \\
& \left(N^{\prime}, \psi^{\prime}, f^{\prime}\right) \preceq(N, \psi, f)
\end{aligned}
$$

This is an equivalence relation on $\mathcal{F}$. For every element $(N, \psi, f)$ of $\mathcal{F}$, we denote by $[N, \psi, f]$ the equivalence class of $(N, \psi, f)$. Let $\overline{\mathcal{F}}$ be the quotient set $\mathcal{F} / \simeq$. The relation $\preceq$ induces a partial order on $\overline{\mathcal{F}}$. Observe that $[M, \phi, I d]$ is a minimum, i.e. it minorates every element of $\overline{\mathcal{F}}$.

We are going to show that every totally ordered subset in $(\overline{\mathcal{F}}, \preceq)$ has an upper bound in $\overline{\mathcal{F}}$. Then, by Zorn's Lemma, $\overline{\mathcal{F}}$ contains at least one maximal element for the order relation $\preceq$. Any representative in $\mathcal{F}$ of this element will be a maximal conformally flat extension of $M$.

Let $\left\{\left[M_{i}, \psi_{i}, f_{i}\right]\right\}_{i \in I}$ be a totally ordered subset of $\overline{\mathcal{F}}$. We can assume, without loss of generality, that $I$ contains a minimal element, denoted by 0 , such that $M_{0}=M, f_{0}=I d$ and $\psi_{0}=\phi$. If $i<j$, let $h_{i, j}: M_{i} \rightarrow M_{j}$ be the unique Cauchy-embedding such that $h_{i, j} \circ \psi_{i}=\psi_{j}$. By Lemma 3.4 $h_{i, k} \circ h_{k, j}=h_{i, j}$ and $h_{i, i}=i d$ for all $i \leq j \leq k$; moreover $h_{0, i}=f_{i}$, for all $i \in I$.

Let

$$
\mathcal{M}:=\bigsqcup_{i \in I} M_{i}
$$

We consider the following relation on $\mathcal{M}$ : given $x \in M_{i}$ and $y \in M_{j}$ then

$$
x \sim y \Leftrightarrow \begin{cases}i \leq j & h_{i, j}(x)=y \\ \text { or } & \\ j<i & h_{j, i}(y)=x\end{cases}
$$

The foregoing shows that $\sim$ is an equivalence relation. Let

$$
\bar{M}:=\mathcal{M} / \sim
$$


equipped with the quotient topology. We want to show that $\bar{M}$ is an element of $\mathcal{F}$.

Let $p_{i}: M_{i} \rightarrow \bar{M}$ the composition of the inclusion $M_{i} \subset \mathcal{M}$ with projection to the quotient $\pi: \mathcal{M} \rightarrow \bar{M}$. If $i<j$ we have $p_{i}\left(M_{i}\right) \subset p_{j}\left(M_{j}\right)$.

Lemma 3.6. Every $p_{i}$ is a homeomorphism onto its image.

Proof. By definition of quotient topology, $p_{i}$ is continuous. Let $U \subset M_{i}$ be an open set, then $p_{i}(U)$ is open in $\bar{M}$ if and only if $\pi^{-1}\left(p_{i}(U)\right)$ is open in $\mathcal{M}$. We have:

$$
\pi^{-1}\left(p_{i}\left(M_{i}\right)\right)=\left(\bigsqcup_{j \in I: i<j} h_{i, j}\left(M_{i}\right)\right) \sqcup\left(\bigsqcup_{j \in I: j \leq i} M_{j}\right)
$$

Moreover every $h_{i, j}\left(M_{i}\right)$ is open in $M_{j}$ because $h_{i, j}$ is an embedding. It is then clear that $\pi^{-1}\left(p_{i}(U)\right)$ is open in $\mathcal{M}$.

The delicate point in the proof that $\bar{M}$ lies in $\mathcal{F}$ is to show is that $\bar{M}$ is a manifold, in particular that it is a second-countable topological space. This is not trivial because $I$ is not countable in general.

Proposition 3.7. $\bar{M}$ is a conformally flat space-time.

Proof. According to Lemma 3.6 every point $p$ of $\bar{M}$ is contained in a neighborhood homeomorphic to $M_{i}$ for some $i$, hence $p$ has a neighborhood homeomorphic to $\mathbb{R}^{n}$. Moreover, every pair of points $q_{1}, q_{2}$ of $\bar{M}$ is contained in the same $p_{i}\left(M_{i}\right)$ for some $i \in I$. By Lemma 3.6 $q_{1}, q_{2}$ have two disjoint neighborhoods, so the space $\bar{M}$ is a Hausdorff topological space. To conclude the proof we have to show that $\bar{M}$ is second-countable and that it is endowed with a conformally flat Lorentzian metric.

We first consider the case where $M$ is simply connected. According to Theorem 2.11 the topology of any globally hyperbolic space-time is determined by the topology of its Cauchy hypersurfaces. Hence, in our case, every space-time in $\mathcal{F}$ is simply connected. Therefore for every $M_{i}$ in $\mathcal{F}$ there is a developing map $d_{i}: M_{i} \rightarrow \operatorname{Ein}_{1, n}$.

Let $i<j$ be two elements of $I$. The map $d_{j} \circ h_{i, j}: M_{i} \rightarrow \widetilde{\operatorname{Ein}}_{1, n}$ is another developing map for $M_{i}$. Therefore there is a unique $g_{i, j}$ in $O(2, n)$ such that $d_{j} \circ h_{i, j}=g_{i, j} \circ d_{i}$. We can then define a map $\bar{d}: \bar{M} \rightarrow \operatorname{Ein}_{1, n}$ by:

$$
\bar{d}(x)= \begin{cases}d_{0} \circ p_{0}^{-1}(x) & \text { if } x \in p_{0}(M) \\ \left(g_{0, i}\right)^{-1} \circ d_{i} \circ p_{i}^{-1}(x) & \text { if } x \in p_{i}\left(M_{i}\right)\end{cases}
$$

First we have to show that $\bar{d}$ is a well-defined map. Let $x$ be a point in $\bar{M} ; x$ is contained in $p_{i}\left(M_{i}\right)$ for some $i$. Let $j$ such that $i<j$; then $p_{i}\left(M_{i}\right) \subset p_{j}\left(M_{j}\right)$. Since $h_{0, j}=h_{i, j} \circ h_{0, i}$ we obtain $g_{0, j}=g_{i, j} \circ g_{0, i}$. Then

$$
\begin{aligned}
\left(g_{0, j}\right)^{-1} \circ d_{j} \circ p_{j}^{-1}(x) & =\left(g_{i, j} \circ g_{0, i}\right)^{-1} \circ d_{j} \circ p_{j}^{-1}(x) \\
& =\left(g_{0, i}\right)^{-1} \circ\left(g_{i, j}\right)^{-1} \circ d_{j} \circ p_{j}^{-1}(x) \\
& =\left(g_{0, i}\right)^{-1} \circ\left(g_{i, j}\right)^{-1} \circ d_{j} \circ h_{i, j} \circ\left(p_{i}\right)^{-1}(x) \\
& =\left(g_{0, i}\right)^{-1} \circ d_{i} \circ p_{i}^{-1}(x)
\end{aligned}
$$


the last equality being true because $p_{j}=\left(h_{i, j}\right)^{-1} \circ p_{i}$ and $\left(g_{i, j}\right)^{-1} \circ d_{j} \circ$ $h_{i, j}=d_{i}$.

The map $\bar{d}$ is well-defined and, by construction, a local homeomorphism. The pull-back by $\bar{d}$ of any Riemannian metric over $\operatorname{Ein}_{1, n}$ defines a Riemannian metric over $\bar{M}$ : then the open balls for this metric on $\bar{M}$ give a countable basis for the topology, so $\bar{M}$ is a second-countable topological space. Moreover the map $\bar{d}$ defines a conformally flat Lorentzian structure on $\bar{M}$. Since the map $h_{i, j}$ preserves the orientation and the chronological orientation, the map $g_{j, i}$ is an element of $O_{0}(2, n)$, for all $i \leq j$. It implies that $\bar{M}$ is chronologically oriented. We have proved that, when $M$ is simply connected, $\bar{M}$ is a conformally flat space-time.

We can now show the theorem in the general case, when $M$ is not necessarily simply connected. First we prove that the universal covering $\tilde{M}$ of $M$ has a naturally defined space-time structure: the lifting by the covering map $\pi: \widetilde{M} \rightarrow M$ of the causal structure of $M$.

Lemma 3.8. Let $M$ be a globally hyperbolic space-time and let $S$ be a Cauchy hypersurface of $M$. Let $\widetilde{M}$ be the universal covering of $M$. Then every lift $\widetilde{S}$ of $S$ is a Cauchy hypersurface of $\widetilde{M}$.

Proof. The covering map $\pi: \widetilde{M} \underset{\sim}{\rightarrow} M$ is a local diffeomorphism which preserves the causal structures of $\widetilde{M}$ and $M$. In particular, causal curves in $\widetilde{M}$ are precisely lifts of causal curves in $M$. It follows that $\tilde{S}$ is a locally achronal embedded hypersurface of $\widetilde{M}$. If $c$ is a timelike curve intersecting $\tilde{S}$ twice, then the projection $\pi \circ c$ intersects $S$ twice: it is impossible since $S$ is achronal; therefore, $\tilde{S}$ is also achronal in $\widetilde{M}$. Moreover $\tilde{S}$ is edgeless because this is a local property.

Let $\alpha: \mathbb{R} \rightarrow \widetilde{M}$ an inextensible causal curve of $\widetilde{M}$. The map $\pi \circ \alpha$ is an immersion such that the image of every vector which is tangent to $\alpha$ is a causal vector of $M$. By Definition 2.3, $M$ is strongly causal, then $\pi \circ \alpha$ must be injective and not self-accumulating. This means that $\pi \circ \alpha$ is an embedding. The map $\pi \circ \alpha$ is then an inextensible causal curve of $M$. Since $S$ is a Cauchy hypersurface of $M$, the curve $\pi \circ \alpha$ intersects $S$. This implies that $\alpha$ intersects $\tilde{S}$, hence $\tilde{S}$ is edgeless and a Cauchy hypersurface of $\widetilde{M}$.

Let $\tilde{h}_{i, j}: \tilde{M}_{i} \rightarrow \tilde{M}_{j}$ be the conformal embedding which lifts the map $h_{i, j}$, where $\tilde{M}_{i}, \tilde{M}_{j}$ are the universal coverings of $M_{i}, M_{j} \in \mathcal{F}$ with $i<j$. According to Lemma 3.8 the lift of every Cauchy hypersurface $S_{i}$ of $M_{i}$ is a Cauchy hypersurface $\tilde{S}_{i}$ of $\tilde{M}_{i}$. Therefore, the maps $h_{i, j}$ are conformal Cauchy-embeddings. The following diagram commutes:

$$
\begin{array}{ccc}
\tilde{\Sigma} \stackrel{\tilde{\psi}_{0}}{\longrightarrow} \tilde{M}_{0} \stackrel{\tilde{h}_{0, i}}{\longrightarrow} \tilde{M}_{i} \stackrel{\tilde{h}_{i, j}}{\longrightarrow} \tilde{M}_{j} \longrightarrow \cdots \\
q_{0} \downarrow \quad q_{i} \downarrow \quad q_{j} \downarrow \\
\Sigma \stackrel{\psi_{0}}{\longrightarrow} M_{0} \stackrel{h_{0, i}}{\longrightarrow} M_{i} \stackrel{h_{i, j}}{\longrightarrow} M_{j} \longrightarrow \cdots
\end{array}
$$

where $q_{k}$ are the covering maps. By the same process used in the definition of $\bar{M}$ we can define a space-time $\bar{M}$ which now is second-contable and equipped with a naturally defined conformally flat space-time structure. 
Let $\bar{d}$ be the developing map of $\bar{M}$ and let $\tilde{p}_{i}: \tilde{M}_{i} \rightarrow \overline{\tilde{M}}$ be the continuous and open maps given by Lemma 3.6. We define the map $p: \bar{M} \rightarrow \bar{M}$ as

$$
p(x)=p_{i} \circ q_{i} \circ \tilde{p}_{i}^{-1}(x)
$$

where $x \in \tilde{p}_{i}\left(\tilde{M}_{i}\right)$. This definition is independent to the choice of the map $\tilde{p}_{i}$. Indeed, if $i<j$, for every $x$ in $\tilde{p}_{i}\left(\tilde{M}_{i}\right)$ we have $\tilde{p}_{i}=\tilde{p}_{j} \circ \tilde{h}_{i, j}$ and $p_{i} \circ q_{i}=p_{j} \circ q_{j} \circ \tilde{h}_{i, j}$, which implies:

$$
p_{i} \circ q_{i} \circ \tilde{p}_{i}^{-1}(x)=p_{j} \circ q_{j} \circ \tilde{p}_{j}^{-1}(x) .
$$

The map $p$ is a local diffeomorphism since it is the composition of local diffeomorphisms. We want to show that $p$ is a covering map. Let $\Gamma:=$ $\pi_{1}(\Sigma)$. The group $\Gamma$ acts over $\tilde{M}_{i}$ in such a way that for every $\gamma$ in $\Gamma$, $q_{i} \circ \gamma=q_{i}$ and for every $i, j$ in $I \tilde{h}_{i, j} \circ \gamma=\gamma \circ \tilde{h}_{i, j}$. Then we can define an action of $\Gamma$ over $\overline{\tilde{M}}$ by:

$$
\gamma(x):=\tilde{p}_{i} \circ \gamma \circ \tilde{p}_{i}^{-1}(x)
$$

where $\forall x \in \tilde{p}_{i}\left(\tilde{M}_{i}\right)$. This action is well-defined: if $i<j$, then

$$
\tilde{p}_{i} \circ \gamma \circ \tilde{p}_{i}^{-1}(x)=\tilde{p}_{j} \circ \tilde{h}_{i, j} \circ \gamma \circ \tilde{h}_{i, j}^{-1} \circ \tilde{p}_{j}^{-1}(x)=\tilde{p}_{j} \circ \gamma \circ \tilde{p}_{j}^{-1}(x) .
$$

By construction, $p \circ \gamma=p$. Moreover, for every $x, y$ in $\bar{M}$ we have $p(x)=p(y)$ if and only if there is an element $\gamma$ of $\Gamma$ such that $x=\gamma(y)$. This action is proper and discontinuous since $\Gamma$ acts properly and discontinuously over every $\tilde{p}_{i}\left(\tilde{M}_{i}\right)$. Then $\bar{M}$ is the quotient of $\overline{\tilde{M}}$ by $\Gamma$ and $p$ is the projection to the quotient.

Since $\bar{M}$ is the quotient of a second-countable manifold by a proper and discontinuous action, it is also a second-countable manifold. Moreover, since the maps $d_{i}$ are equivariant for the action of $\Gamma$ over $\tilde{M}_{i}$, the local diffeomorphism $\bar{d}$ is also equivariant for the action of $\Gamma$. Then there is a well-defined conformally flat space-time structure over $\bar{M}$.

Now that we have shown that $\bar{M}$ is a space-time, we can study his causal structure. We want to prove that it is globally hyperbolic with Cauchy hypersurface $p_{0}\left(S_{0}\right)$.

Lemma 3.9. Every $p_{i}\left(M_{i}\right)$ is causally convex inside $\bar{M}$.

Proof. Let $c:[0,1] \rightarrow \bar{M}$ be a causal curve between two points of $p_{i}\left(M_{i}\right)$. The set $c([0,1])$ is a compact subset of $\bar{M}$. Since $\bar{M}$ is the growing union of the sets $p_{k}\left(M_{k}\right)$ and since $I$ is a totally ordered set, there exists $j \in I$ such that $c([0,1]) \subset p_{j}\left(M_{j}\right)$. We suppose $i \leq j$ (up to replacing $j$ by $i$ if $j<i)$. The open set $p_{i}\left(M_{i}\right)$ is causally convex in $p_{j}\left(M_{j}\right)$ since $h_{i, j}\left(M_{i}\right)$ is causally convex in $M_{j}$ and because of Lemma 3.1 (Recall that $p_{j}$ is a conformal embedding.) Thus the image of $c$ is contained in $p_{i}\left(M_{i}\right)$.

The image $p_{i}\left(S_{i}\right)$ does not depend on $i$, it is a spacelike hypersurface inside $\bar{M}$ that we denote by $\bar{S}$.

Lemma 3.10. $\bar{S}$ is a Cauchy hypersurface of $\bar{M}$. In particular, $\bar{M}$ is globally hyperbolic. 
Proof. Let $c$ be a timelike curve between two points of $\bar{S}$. Since $c$ is compact, there exists $i \in I$ such that $c$ is contained in $p_{i}\left(M_{i}\right)$. This contradicts the fact that $\bar{S}=p_{i}\left(S_{i}\right)$ is achronal in every $p_{i}\left(M_{i}\right)$. Thus $\bar{S}$ is achronal.

The hypersurface $\bar{S}$ is edgeless because this is a local property and $\bar{S}$ is edgeless in every $p_{i}\left(M_{i}\right)$. Every point $p$ of $\bar{M}$ is contained in $p_{i}\left(M_{i}\right)$ for some $i \in I$, which is globally hyperbolic with Cauchy hypersurface $\tilde{S}$. Hence $p$ is contained in the Cauchy development of $\bar{S}$ in $\bar{M}$. This proves that $\bar{S}$ is a Cauchy hypersurface of $\bar{M}$.

We have proved that $\bar{M}$ is an element of $\mathcal{F}$. Moreover, for every $i \in I$, the map $p_{i}$ is a Cauchy-embedding of $M_{i}$ into $\bar{M}$. Let $\bar{f}$ be the Cauchyembedding given by $p_{0}$ and $\bar{\psi}$ the composition of $\phi$ and the restriction of $\bar{f}$ to $S$, then he element $[\bar{M}, \bar{\psi}, \bar{f}]$ of $\overline{\mathcal{F}}$ and is an upper bound for the totally ordered set $\left\{\left[M_{i}, \psi_{i}, f_{i}\right]\right\}_{i \in I}$. By Zorn's Lemma, $\overline{\mathcal{F}}$ has at least one maximal element. It follows that any representative in $\mathcal{F}$ of this maximal element is a maximal conformally flat extension of $M$.

Now we have to prove the uniqueness of the maximal extension. That is: up to conformal diffeomorphism there is a unique maximal element in $\overline{\mathcal{F}}$.

Let $F_{1}: M_{0} \rightarrow M_{1}^{\max }$ and $F_{2}: M_{0} \rightarrow M_{1}^{\max }$ be two Cauchy-embeddings of $M_{0}$ in two maximal extensions: we want to prove that $M_{1}^{\max }$ and $M_{2}^{\max }$ are conformally equivalent.

Let $\mathcal{H}$ be the set of quadruples $\left(M, f, g_{1}, g_{2}\right)$ such that $f: M_{0} \rightarrow M$, $g_{1}: M \rightarrow M_{1}^{\max }$ and $g_{2}: M \rightarrow M_{2}^{\max }$ are Cauchy-embeddings, where $F_{i}=g_{i} \circ f$ for $i=1,2$. Over $\mathcal{H}$ we define the relation

$$
\begin{aligned}
\left(M, f, g_{1}, g_{2}\right) \preceq\left(M^{\prime}, f^{\prime}, g_{1}^{\prime}, g_{2}^{\prime}\right) \Longleftrightarrow & \exists \Phi: M \rightarrow M^{\prime} \text { Cauchy-embedding } \\
& \text { such that } f^{\prime}=\Phi \circ f \text { and } g_{i}=g_{i}^{\prime} \circ \Phi
\end{aligned}
$$

where $i=1,2$. This relation leads to a partial order over the quotient $\overline{\mathcal{H}}$ by the equivalence relation, which identifies two quadruples $\left(M, f, g_{1}, g_{2}\right)$ and $\left(M^{\prime}, f^{\prime}, g_{1}^{\prime}, g_{2}^{\prime}\right)$ if the Cauchy-embedding $\Phi$ is surjective. The elements of $\overline{\mathcal{H}}$ are denoted by $\left[M, f, g_{1}, g_{2}\right]$.

Just like in the proof of the maximal extension's existence, we prove that $(\overline{\mathcal{H}}, \preceq)$ is inductive: given a totally ordered set $\left\{\left[M^{k}, f^{k}, g_{1}^{k}, g_{2}^{k}\right]\right\}_{k \in I}$ of $\overline{\mathcal{H}}$, we consider the quotient $\bar{M}$ of the disjoint union of every $M^{k}$ the equivalence relation which identifies every $\Phi_{k, l}(x)$ with $x$ if $k<l$, where $\Phi_{k, l}(x)$ is the unique Cauchy-embedding such that $f^{l}=\Phi_{k, l} \circ f^{k}$ and $g_{i}^{k}=$ $g_{i}^{i} \circ \Phi_{k, l}$.

The maps $g_{1}^{k}, g_{2}^{k}$ and $f_{k}$ are compatible with this relation and then they induce, at the quotient level, the Cauchy-embeddings $\bar{g}_{i}: \bar{M} \rightarrow M_{i}^{\max }$, where $i=1,2$, and $\bar{f}: M_{0} \rightarrow \bar{M}$. As in the existence proof, one shows that this quotient is a conformally globally hyperbolic space-time, which gives an upper bound for the set $\left\{\left[M^{k}, f^{k}, g_{1}^{k}, g_{2}^{k}\right]\right\}_{k \in I}$ (see Figure 21).

Once more, Zorn's Lemma implies that $\overline{\mathcal{H}}$ contains a maximal element, denoted by $\left[\bar{M}, \bar{f}, \bar{g}_{1}, \bar{g}_{2}\right]$. 


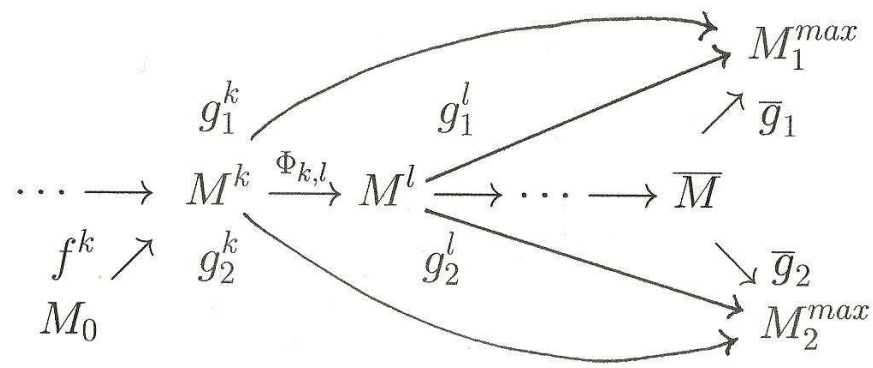

Figure 2: A totally ordered set in $\overline{\mathcal{H}}$ with the maximal element $\left[\bar{M}, \bar{f}, \bar{g}_{1}, \bar{g}_{2}\right]$.

Let $\mathcal{M}$ be the quotient of the disjoint union $M_{1}^{\max } \bigsqcup M_{2}^{\max }$ by the relation which identifies $\bar{g}_{1}(x)$ with $\bar{g}_{2}(x)$ for every $x \in \bar{M}$. The projections $\pi_{i}$ : $M_{i}^{\max } \rightarrow \mathcal{M}$ are conformal embeddings, and every point of $\mathcal{M}$ has a neighborhood homeomorphic to $\mathbb{R}^{n}$. To prove that $\mathcal{M}$ is a manifold, the key point that we have to check is:

Lemma 3.11. $\mathcal{M}$ is Hausdorff.

Proof. Let $(x, y)$ be a pair of points in $M_{1}^{\max } \times M_{2}^{\max }$ such that every neighborhood of $\pi_{1}(x)$ intersects every neighborhood of $\pi_{2}(y)$. The points $x$ and $y$ are contained in respectively $\partial \bar{g}_{1}(\bar{M})$ and $\partial \bar{g}_{2}(\bar{M})$. Let

$$
S_{i}^{+}:=\partial^{+} \bar{g}_{i}(\bar{M}) \text { and } S_{i}^{-}:=\partial^{-} \bar{g}_{i}(\bar{M})
$$

be respectively the past and future boundary in $M_{i}^{\max }$ of the image of $\bar{M}$ by the embedding $\bar{g}_{i}$, for $i=1,2$. According to Lemma 3.5. $S_{i}^{+}$and $S_{i}^{-}$are closed, achronal, edgeless subsets of $M_{i}^{\max }$, and $S_{i}^{+} \cap S_{i}^{-}=\emptyset$, for $i=1,2$. Up to time reversal, we can assume, without loss of generality, that $x \in S_{1}^{+}$. Let $\Sigma_{1}$ and $\Sigma_{2}$ be the Cauchy hypersurfaces respectively of $M_{1}^{\max }$ and $M_{2}^{\max }$ defined by:

$$
\Sigma_{1}:=\bar{g}_{1} \circ \bar{f}\left(S_{0}\right) \text { and } \Sigma_{2}:=\bar{g}_{2} \circ \bar{f}\left(S_{0}\right) .
$$

Let $U$ be a (connected) neighborhood of $x$ in $M_{1}^{\max }$ such that $U \cap \Sigma_{1}=\emptyset$ and $U \backslash S_{1}^{+}$is the disjoint union of the two connected open sets : $U \cap \bar{g}_{1}(\bar{M})$ and $U \cap I^{+}\left(S_{1}^{+}\right)$. Then $U$ is contained in $I^{+}\left(\Sigma_{1}\right)$. The image $U \cap \bar{g}_{1}(\bar{M})$ by the conformal, time-preserving diffeomorphism $\bar{g}_{2} \circ \bar{g}_{1}^{-1}$ is then contained in $I^{+}\left(\Sigma_{2}\right)$. Thus $y \in S_{2}^{+}$.

Since by hypothesis every neighborhood of $\pi_{1}(x)$ intersects every neighborhood of $\pi_{2}(y)$, there exists a neighborhood $U^{\prime}$ of $y$ in $M_{2}^{\max }$ such that 
$\bar{g}_{2} \circ \bar{g}_{1}^{-1}\left(U \cap \bar{g}_{1}(\bar{M})\right)=U^{\prime} \cap \bar{g}_{2}(\bar{M})$.

Key fact: by Liouville's Theorem 2.23 there exists a neighborhood $V$ of y and a conformal diffeomorphism $f: U \rightarrow V$ which is equal to $\bar{g}_{2} \circ \bar{g}_{1}^{-1}$ over $U \cap \bar{g}_{1}(\bar{M})$.

First assume that the future Cauchy development of $D_{1}^{+}$of the achronal set $S_{1}^{+} \cap U$ in $U$ is not empty. Since the open sets $U$ and $V$ are conformally equivalent (by the map $f$ ), they have the same causal structure. In particular, the future Cauchy development $D_{2}^{+}$of $S_{2}^{+} \cap U_{2}$ in $V$ is not empty too. Let

$$
F: \bar{g}_{1}(\bar{M}) \cup D_{1}^{+} \longrightarrow \bar{g}_{2}(\bar{M}) \cup D_{2}^{+}
$$

be the conformal diffeomorphism defined by:

$$
F(z):= \begin{cases}\bar{g}_{2} \circ \bar{g}_{1}^{-1}(z) & \text { if } z \in \bar{g}_{1}(\bar{M}) \\ f(z) & \text { if } z \in D_{1}^{+}\end{cases}
$$

Thus, the two element of the set $\overline{\mathcal{H}}$ given by

$$
\left[\bar{g}_{1}(\bar{M}) \cup D_{1}^{+}, \bar{g}_{1} \circ \bar{f}, i d, F\right] \quad \text { and } \quad\left[\bar{g}_{2}(\bar{M}) \cup D_{2}^{+}, \bar{g}_{2} \circ \bar{f}, F^{-1}, i d\right]
$$

are strictly greater then $\left[\bar{M}, \bar{f}, \bar{g}_{1}, \bar{g}_{2}\right]$. This contradicts the maximality of $\left[\bar{M}, \bar{f}, \bar{g}_{1}, \bar{g}_{2}\right]$.

Therefore, the future Cauchy development of $S_{1}^{+} \cap U$ in $U$ is empty.

According to Lemma 2.9, there is a lightlike past geodesic $c$ starting from $x$ which is contained in $S_{1}^{+} \cap U$ at least in a neighborhood of $x$. Let $r_{1}:\left[0, a\left[\rightarrow M_{1}^{\max }\right.\right.$ be an inextensible lightlike past geodesic containing $c$. The curve $r_{1}$ intersects the Cauchy hypersurface $\Sigma_{0}$ of $M_{1}^{\max }$, in a point $r_{1}\left(T_{0}\right)$. Since $\Sigma_{0}$ is contained in $\bar{g}_{1}(\bar{M})$ and $S_{1}^{+}$is achronal and closed, there exists a $T \in[0, a[$ such that

$$
T:=\max \left\{t \in \left[0, a\left[: r_{1}(t) \in S_{1}^{+}\right\}\right.\right.
$$

We have $0<T<T_{0}<a$ and $\left.\left.c(] T, T_{0}\right]\right)$ is contained in $\bar{g}_{1}(\bar{M})$.

Let $\left\{U_{1}\right\}_{i=0, \ldots, m}$ be a finite covering of the compact set $r_{1}([0, T])$ such that $U_{i} \cap \bar{g}_{1}(\bar{M})$ and $U_{i} \cap I^{+}\left(S_{1}^{+}\right)$are connected for all $i=1, \ldots, m$. We can suppose $U_{0}=U$. Again, by Liouville's Theorem there exists a finite sequence $\left\{V_{1}\right\}_{i=0, \ldots, m}$ of open sets and conformal diffeomorphisms $f_{i}: U_{i} \rightarrow V_{i}$, for $i=0, \ldots, m$ such that $f_{0}=f, V_{0}=V$ and $f_{i}$ is equal to $\bar{g}_{2} \circ \bar{g}_{1}^{-1}$ over $U_{i} \cap \bar{g}_{1}(\bar{M})$.

The isometries $f_{i}$ glue together to give a map $F: \bigcup_{i=0}^{n} U_{i} \rightarrow \bigcup_{i=0}^{n} V_{i}$. Then, for every $t \in[0, T]$, the points $\pi_{1}\left(r_{1}(t)\right)$ and $\pi_{2}\left(F \circ r_{1}(t)\right)$ of $\mathcal{M}$ have no disjointed neighborhoods.

Now we can again apply Lemma 2.9. to find a past lightlike geodesic $\gamma$ starting from $r_{1}(T)$ and contained in $S_{1}^{+}$for a little while. According to Proposition 2.2. $\gamma$ is contained inside $r_{1}$, because $S_{1}^{+}$is achronal. But if $\gamma$ is contained in $r_{1}$ then $r_{1}(T+\varepsilon) \in S_{1}^{+}$for some $\varepsilon>0$, and this contradicts the definition of $T$. The Lemma is proven. 
Let $\Sigma:=\pi_{1} \circ \bar{g}_{1} \circ \bar{f}(S)$. Observe that $\Sigma$ is equal to $\pi_{2} \circ \bar{g}_{2} \circ \bar{f}(S)$.

Lemma 3.12. $\mathcal{M}$ is globally hyperbolic with Cauchy hypersurface $\Sigma$.

Proof. Let $c: \mathbb{R} \rightarrow \mathcal{M}$ be an inextensible causal curve. Suppose that $c$ does not intersect $\pi_{1} \circ \bar{g}_{1}(\bar{M})=\pi_{2} \circ \bar{g}_{2}(\bar{M})$. Then, since $\mathbb{R}$ is connected, $c$ is contained in either $\pi_{1}\left(M_{1}^{\max }\right) \backslash \pi_{1} \circ \bar{g}_{1}(\bar{M})$ or in $\pi_{2}\left(M_{2}^{\max }\right) \backslash \pi_{1} \circ \bar{g}_{1}(\bar{M})$. Switching the indices if necessary, we can suppose that $c$ is contained in $\pi_{1}\left(M_{1}^{\max }\right) \backslash \pi_{1} \circ \bar{g}_{1}(\bar{M})$. Then, there is a causal curve $c^{\prime}: \mathbb{R} \rightarrow M_{1}^{\max }$ such that $c=\pi_{1} \circ c^{\prime}$. Since $c$ is inextensible, so is $c^{\prime}$, but $c^{\prime}$ must intersect $\bar{g}_{1}(\bar{M})$. This is a contradiction.

We have proved that every inextensible causal curve $c$ intersects $\pi_{1} \circ$ $\bar{g}_{1}(\bar{M})$. Every connected component of its intersection with $\pi_{1} \circ \bar{g}_{1}(\bar{M}) \simeq$ $\bar{M}$ is an inextensible causal curve (into $\pi_{1} \circ \bar{g}_{1}(\bar{M})$ ) and thus intersects $\pi_{1} \circ \bar{g}_{1} \circ f_{1}(S)=\Sigma$. Moreover, since $\pi_{1} \circ \bar{g}_{1}(\bar{M})$ is causally convex inside $M_{1}^{\text {max }}$, it is also causally convex inside $\mathcal{M}$, implying that $c \cap \pi_{1} \circ \bar{g}_{1}(\bar{M})$ has only one connected component. We have proved that every inextensible causal curve intersects $\Sigma$. The hypersurface $\Sigma$ is achronal and edgeless in $\mathcal{M}$, so we have proved that $\Sigma$ is a Cauchy hypersurface of $\mathcal{M}$.

As consequence of the proof of Lemma 3.12, the maps $\pi_{i}$ are Cauchyembeddings. The space-time $\mathcal{M}$ is conformally flat because it is covered by conformally flat open subsets given by the images of $\pi_{i} \circ \bar{g}_{i}, i=1,2$. Since, by hypothesis, the space-times $M_{1}^{\max }$ and $M_{2}^{\max }$ are maximal, the Cauchy-embeddings $\pi_{1}$ and $\pi_{2}$ are surjective, and $M_{1}^{\max }$ and $M_{2}^{\max }$ are conformally equivalent. The proof of Theorem 1.7 is complete.

Remark 3.13. Until now we have restricted ourselves to the case of conformally flat space-times. If we drop this restriction and define $\mathcal{F}$ as the set of triples $(N, \psi, f)$, where $\psi$ and $f$ are exactly as before, but where $N$ is only globally hyperbolic and not necessarily conformally flat, most of the arguments in the proof above still apply. Given a totally ordered set $\left\{\left(M_{i}, S_{i}\right)\right\}_{i \in I}$ we can still define a topological space $\bar{M}$, candidate to be a maximal conformal extension of $M$.

But if one wants to prove that $\mathcal{M}$ is a manifold, serious troubles arise. The proof of Proposition 3.7 strongly uses the conformally flat structure of the manifolds $M_{i}$. The most delicate point is to prove that $\bar{M}$ has a second-countable basis as a topological space.

Even if one were able to prove the existence of the conformal maximal extension, we have no way to prove uniqueness. In the conformally flat case, uniqueness follows from Liouville's theorem for conformally flat manifolds of dimension $\geq 3$.

However, we still don't know any examples admitting different maximal conformal extensions. Actually, all known examples of conformally flat maximal space-times are also conformal maximal. So it seems interesting to investigate if, despite these difficulties, there is a well-defined notion of maximal conformal extension, in the category of conformal globally hyperbolic space-times. 


\section{Complete $C_{0}$-maximal space-times}

We can easily prove that $\widetilde{\operatorname{Ein}}_{1, n}$ is $C_{0}$-maximal. The proof goes as follows. Let $f: \widetilde{\operatorname{Ein}}_{1, n} \rightarrow N$ be a Cauchy embedding of $\widetilde{\operatorname{Ein}}_{1, n}$. According to Proposition 2.12, $N$ is simply connected. But, since $N$ is conformally flat, there exists a developing map $D: N \rightarrow \widetilde{\operatorname{Ein}}_{1, n}$. The composition $D \circ f: \widetilde{\operatorname{Ein}}_{1, n} \rightarrow \widetilde{\operatorname{Ein}}_{1, n}$ preserves the conformal structure of $\widetilde{\operatorname{Ein}}_{1, n}$, and by Liouville's Theorem $D \circ f$ is an element of $\widetilde{O_{0}}(2, n+1)$. Hence, $f$ is surjective, and $\widetilde{\operatorname{Ein}}_{1, n}$ is $C_{0}$-maximal. The following lemma is well known in the literature. (See for example [18] Lemma 1 p. 7 for an idea of the proof.)

Lemma 4.1. Let $f:(S, g) \rightarrow\left(S^{\prime}, g^{\prime}\right)$ be a local diffeomorphism between two Riemannian manifolds. Suppose that $(S, g)$ is complete and that $g(\cdot, \cdot) \leq f^{*} g^{\prime}(\cdot, \cdot)$. Then $f$ is a covering map.

Lemma 4.2. Let $M$ be a causal, conformally flat, simply connected spacetime. Then the developing map $D: M \rightarrow \widehat{\operatorname{Ein}}_{1, n}$ restricted to any causal curve of $M$ is injective.

Proof. Let $T: \widetilde{\text { Ein }} \rightarrow \mathbb{R}$ be any time function on $\widetilde{\text { Ein }}$. The restriction of $T \circ D$ to any future causal curve of $M$ is increasing, hence injective. The lemma is proved.

Now we can prove Theorem 1.8, which can be restated as follows:

Theorem 4.3. For $n \geq 2, \widetilde{\operatorname{Ein}}_{1, n}$ is the only conformally flat, simply connected, Cauchy-compact space-time which is $C_{0}$-maximal.

Proof. Let $M$ be a conformally flat simply connected $C_{0}$-maximal spacetime and let $\Sigma \subset M$ be a compact, spacelike Cauchy hypersurface of $M$. According to Corollary $2.12 M$ is diffeomorphic to the product $\Sigma \times \mathbb{R}$, and $\Sigma$ is simply connected.

Let $D: M \rightarrow \operatorname{Ein}_{1, n}$ be the developing map of the conformally flat structure on $M$. Consider the decomposition $\operatorname{Ein}_{1, n} \simeq\left(\mathbb{S}^{n} \times \mathbb{R},\left[d \sigma^{2}-\right.\right.$ $\left.d t^{2}\right]$ ), described in Section 2.2. The pull-back of the metric $d \sigma^{2}-d t^{2}$ by $D$ is a Lorentzian metric over $M$ which defines a Riemannian metric $g_{0}$ on $\Sigma$. The metric $g_{0}$ is complete because $\Sigma$ is compact.

Let $\pi: \operatorname{Ein}_{1, n} \simeq \mathbb{S}^{n} \times \mathbb{R} \rightarrow \mathbb{S}^{n}$ be the projection on the first factor. This is a bundle whose fibers are the orbits of the timelike vector field $\partial_{t}$. The main observation is that $\pi^{*} d \sigma=d \sigma \geq d \sigma-d t^{2}$ which implies that $d \pi$ expands the lengths. Since $\left.D\right|_{\Sigma}$ is a local isometry, $d\left(\left.\pi \circ D\right|_{\Sigma}\right)$ also expands the lengths. Explicitly, for all $X$ in $T_{x} \Sigma \subset T_{x} M$ we have :

$$
g_{0}(X, X)=g\left(d_{x} D X, d_{x} D X\right)=d \sigma(Y, Y)-|a|^{2} \leq d \sigma(Y, Y)
$$

where $Y$ is the component of $X$ which is orthogonal to the orbit of $\partial_{t}$ and $a$ the tangent one.

According to Lemma 4.1 the map $\left.\pi \circ D\right|_{\Sigma}: \Sigma \rightarrow \mathbb{S}^{n}$ is a covering map. Since $\mathbb{S}^{n}$ is simply connected, $\left.\pi \circ D\right|_{\Sigma}$ is a diffeomorphism. Therefore the embedded hypersurface $D(\Sigma)$ intersects each orbit of $\partial_{t}$ at exactly one point. The pull-back on $M$ by $D$ of the vector field $\partial_{t}$ of $\operatorname{Ein}_{1, n}$ is 
a timelike vector field $T$. By Lemma 4.2 the map $D$ is injective along the orbits of $T$. Since $\Sigma$ is a Cauchy hypersurface of $M$, every orbit of $T$ intersects $\Sigma$ at exactly one point. Let $p, q \in M, p \neq q$, such that $D(p)=D(q)$ and let $c_{p}, c_{q}$ be the two orbits of $T$, respectively through $p$ and $q$. These orbits intersect $\Sigma$ at two points $\bar{p}$ and $\bar{q}$, obviously we have $\bar{p} \neq \bar{q}$. Since $D$ is a local diffeomorphism, $D\left(c_{p}\right)=D\left(c_{q}\right)$ and it is contained in the orbit of $\partial_{t}$ through the point $D(p)=D(q)$, which intersects $D(\Sigma)$ at a unique point $x$. Thus $x=D(\bar{p})=D(\bar{q})$. This is absurd because $D$ is injective on $\Sigma$. Therefore, $D$ is injective.

Moreover, since the restriction of $D$ to $\Sigma$ is injective, the image $D(\Sigma)$ is a spacelike, compact, edgeless, embedded hypersurface in $\widetilde{\operatorname{Ein}}_{1, n}$. By Proposition 2.13, $D(\Sigma)$ is a Cauchy hypersurface of $\operatorname{Ein}_{1, n}$ and $D$ is a Cauchy embedding. Since $M$ is $C_{0}$-maximal, Theorem 1.7] implies that $D$ is also surjective: $M$ and $\operatorname{Ein}_{1, n}$ are conformally equivalent.

Let $M$ be a conformally flat space-time of dimension $n+1 \geq 3$. Let $D: \widetilde{M} \rightarrow \widetilde{\operatorname{Ein}}_{1, n}$ be the developing map and $\rho: \pi_{1}(M) \rightarrow O_{0}(2, n+$ 1) the holonomy morphism. Lemma 4.2 is related to the problem of finding injectivity domains for $D$. Let $x \in \widetilde{\operatorname{Ein}_{1, n}}$. By Remark 2.21, all the lightlike geodesics starting from $x$ intersect each other only at the conjugate points $\sigma^{k}(x)$ for every $k \in \mathbb{Z}$. Let $\alpha, \beta: \mathbb{R} \rightarrow \widetilde{M}, \alpha(0)=$ $\beta(0)=p$ be two distinct past inextensible lightlike geodesics, such that their images $D \circ \alpha$ and $D \circ \beta$ have an intersection point $z$ other then $D(p)$. Then, if the restriction of $D$ to $\alpha \cup \beta$ is injective, $\alpha$ and $\beta$ necessarily have an intersection point $q \in D^{-1}(z)$. Conversely, if $D(\alpha) \cap D(\beta)=\emptyset$, then the restriction of $D$ to $\alpha \cup \beta$ is injective.

Theorem 4.4. Let $M$ be a globallly hyperbolic conformally flat, $C_{0}$ maximal space-time of dimension $n+1 \geq 3$, and let $D: \widetilde{M} \rightarrow \widehat{\operatorname{Ein}}_{1, n}$ be the developing map. Suppose that there is a lightlike geodesic $\alpha$ in $\widetilde{M}$ whose image by $D$ contains two conjugate points in $\widetilde{\operatorname{Ein}_{1, n}}$. Then $D$ is a diffeomorphism.

Proof. By hypothesis $D \circ \alpha$ contains two conjugate points $x$ and $y$ of $\widetilde{\operatorname{Ein}}_{1, n}$. We can assume, without loss of generality, that $x=\sigma(y)$. Since the restriction of $D$ to $\alpha$ is injective, there are exactly two points $p$ and $q$ of $\alpha$ such that $D(p)=x$ et $D(q)=y$.

Step 1): Every lightlike past inextensible geodesic starting from $p$ meets $q$. Let

$$
\mathcal{N}^{-}(p):=\left\{v \in T_{p} \tilde{M} / v \text { past lightlike }\right\}
$$

On $\mathcal{N}^{-}(p)$ we define the equivalence relation identifying $v \sim w$ if and only if there exists $\lambda \in \mathbb{R}^{+}$such that $v=\lambda w$. Consider the quotient set

$$
\mathcal{S}^{-}(p):=\mathcal{N}^{-}(p) / \sim
$$

This is the set of lightlike past rays starting from $p$. Endowed with the quotient topology, it is homeomorphic to the $(n-1)$-sphere. Let $p_{1}: \mathcal{N}^{-}(p) \rightarrow \mathcal{S}^{-}(p)$ be the projection map.

Let $\mathcal{E}$ the set of $l$ in $\mathcal{S}^{-}(p)$ such that the past inextensible lightlike geodesic 
$\gamma_{l}$ starting from $p$ and tangent to $l$, contains $q$. By hypothesis, $\mathcal{E}$ is not empty, because it contains the direction tangent to $\alpha$. We are going to show that $\mathcal{E}$ is open and closed in $\mathcal{S}^{-}(p)$.

The set $\mathcal{E}$ is open.

Let $l_{0}$ be an element of $\mathcal{E}$. By definition, $\gamma_{l_{0}}$ contains $q$. Let $V$ be an open neighborhood of $y$ and let $W$ be the connected component of $D^{-1}(V)$ containing $q$. We can choose $V$ such that $\left.D\right|_{W}$ is injective.

Since $D$ is continuous and since $\gamma_{l_{0}}$ is past inextensible, $D \circ \gamma_{l_{0}}$ intersects $J^{-}(y) \backslash y$. Let $z$ be a point of $D \circ \gamma_{l_{0}}$ which is in $\left(J^{-}(y) \backslash y\right) \cap V$ and let $q_{0}$ be the unique point of $\gamma_{l_{0}}$ such that $D\left(q_{0}\right)=z$.

Since $J^{+}(y)$ is closed in $V$, we can find an open connected neighborhood $V^{\prime}$ of $z$ such that $V^{\prime} \subset V$ and $V^{\prime} \cap J^{+}(y)=\emptyset$. The connected component $W^{\prime}$ of $D^{-1}\left(V^{\prime}\right)$ contained in $W$ is an open neighborhood of $q_{0}$.

Pick a Lorentzian metric $g$ in the conformal class of $\widetilde{M}$ and let $\exp _{p}$ : $T_{p} \widetilde{M} \rightarrow \widetilde{M}$ be its exponential map at the point $p$. The set $\mathcal{W}:=$ $\exp _{p}^{-1}\left(W^{\prime}\right)$ is an open subset of $T_{p} M$. The subset of $S^{-}(p)$ defined by

$$
\mathcal{U}:=p_{1}\left(\mathcal{W} \cap \mathcal{N}^{-}(p)\right)=\left\{l \in S^{-}(p) / \gamma_{l} \cap W^{\prime} \neq \emptyset\right\}
$$

is then open and contains $l_{0}$.

Let $l$ be in $\mathcal{U}$. The curve $\gamma_{l}$ intersects $W^{\prime}$, therefore $D \circ \gamma_{l}$ intersects $V^{\prime}$. It follows that $D \circ \gamma_{l}$ is a past lightlike geodesic starting at $x$ which intersects $J^{-}(y)$. By Remark 2.21] the curve $D \circ \gamma_{l}$ contains $y$. Since $\left.D\right|_{W}$ is injective, $\gamma_{l}$ contains $q$. This shows that $\mathcal{U}$ is an open neighborhood of $l_{0}$ contained in $\mathcal{E}$ (see Figure ??).

The set $\mathcal{E}$ is closed.

Let $\left\{l_{n}\right\}_{n \in \mathbb{N}}$ be a sequence in $\mathcal{E}$ converging to an element $l \in S^{-}(p)$. According to Lemma 2.4, the sequence $\left\{\gamma_{l_{n}}\right\}_{n \in \mathbb{N}}$ has a limit curve which is a past causal curve $c$ between $p$ and $q$. The fact that $x$ is not temporally related to $y$ implies that $q$ is not temporally related to $p$ (because the image of timelike curves by $D$ are timelike curves). Proposition 2.2 implies that $c$ is a lightlike geodesic. Since $l_{n} \rightarrow l \in S^{-}(p)$, the geodesic $c$ coincides with the geodesic $\gamma_{l}$.

Since $\mathcal{E}$ is open and closed in the (connected) topological sphere $\mathcal{S}^{-}(p)$, it is the entire $\mathcal{S}^{-}(p)$. This prove Step 1 .

Observe that the similar following statement is true: every future lightlike geodesic starting from $q$ contains $p$.

Step 2) $\partial I^{-}(p)$ is a compact Cauchy hypersurface in $\widetilde{M}$.

Let $S$ be the union of all lightlike geodesic segments between $p$ and $q$. By construction, $D(S)=\partial I^{-}(x)$. Let us prove that the restriction of $D$ to $S$ is injective. Let $r, s$ be two points of $S$ with $D(r)=D(s)$. Since any point of $S$ is contained in a lightike geodesic starting from $p$ and ending in $q$, and since $D$ restricted to any causal curve is injective, we have that if $r=p$ (or reps. $r=q$ ) then $s=r=p$ (or reps. $s=r=q$ ). Then we can suppose $r, s \in S \backslash\{p, q\}$. Let $\gamma_{l}, \gamma_{l^{\prime}}$ be the geodesic segments between $p$ and $q$ containing respectively $r$ and $s$. Then $D\left(\gamma_{l}\right)$ and $D\left(\gamma_{l^{\prime}}\right)$ 
are geodesic segments between $x$ and $y$ having the point $D(r)=D(s)$ in common. Moreover, since $\widetilde{\operatorname{Ein}}_{1, n}$ is causal, this point is different to $x$ and $y$. Then the Remark 2.21 implies that the two geodesic $D\left(\gamma_{l}\right)$ and $D\left(\gamma_{l^{\prime}}\right)$ are the same. Since $D$ is a local diffeomorphism, it follows that $l=l^{\prime}$. Since the restriction of $D$ to the causal curve $\gamma_{l}$ is injective, the equality $r=s$ follows.

The set $S$ is achronal in $M$ : if there was a timelike curve between two points of $S$, then the image under $D$ of this curve would be a timelike curve between two points of the achronal subset $D(S)=\partial I^{-}(x)$.

Since $S$ is achronal we have $S \subset \partial I^{-}(p)$. By Proposition 2.2 we have also the inclusion $\partial I^{-}(p) \subset S$. Then $S=\partial I^{-}(p)$. Since $D(S)=\partial I^{-}(x)$ is compact, it follows that $S$ is also compact. Moreover, according to Lemma 2.8 the boundary $\partial I^{-}(p)=S$ of the past subset $I^{-}(p)$ is edgeless.

We have proved that $S$ is a compact, achronal, edgeless subset of $\widetilde{M}$ : by Proposition 2.13, $S$ is a Cauchy hypersurface of $\widetilde{M}$.

Step 3) Since $\widetilde{M}$ admits a compact Cauchy hypersurface, we obtain by Theorem 4.3 that $\widetilde{M}$ is conformally equivalent to $\widetilde{E i n}$.

We can now give the proof of Theorem 4.4 which we restate here for the reader's convenience:

Theorem 1.9. Let $M$ be a conformally flat, globally hyperbolic, $C_{0}$ maximal space-time which has two freely homotopic lightlike geodesics, which are distinct but with the same ends. Then $M$ is a finite quotient of $\widetilde{\operatorname{Ein}}_{1, n}$.

Proof. Since the two geodesic segments are freely homotopic, it is possible to lift them in such a way that the two lifts are two lightlike geodesic segments, $\alpha$ and $\beta$, with the same ends. The image of $\alpha$ by the developing map (and also the image of $\beta$ ) intersects two conjugate points of $\widetilde{\operatorname{Ein}}_{1, n}$. By Theorem 4.4, $\widetilde{M}$ is homeomorphic to $\widetilde{\operatorname{Ein}}_{1, n}$. In particular the lift $\tilde{S}$ of every Cauchy hypersurface $S$ of $M$ is homeomorphic to the sphere $\mathbb{S}^{n}$. The fundamental group of $M$ has to preserve $\tilde{S}$ and it acts properly and discontinuously on $\tilde{S}$. Since $\tilde{S}$ is compact, it follows that $\pi_{1}(M)$ is finite.

\section{References}

[1] Thierry Barbot. Globally hyperbolic flat space-times. J. Geom. Phys., 53(2):123-165, 2005.

[2] Thierry Barbot. Domaines globalement hyperboliques de l'espace de minkowski et de l'espace anti-de sitter. 2010.

[3] Thierry Barbot and Quentin Mérigot. Anosov AdS representations are quasi-Fuchsian. Groups Geom. Dyn., 6(3):441-483, 2012.

[4] John K. Beem, Paul E. Ehrlich, and Kevin L. Easley. Global Lorentzian geometry, volume 202 of Monographs and Textbooks in 
Pure and Applied Mathematics. Marcel Dekker Inc., New York, second edition, 1996.

[5] Antonio N. Bernal and Miguel Sánchez. Globally hyperbolic spacetimes can be defined as 'causal' instead of 'strongly causal'. Classical and Quantum Gravity, 24(3):745, 2007.

[6] Francesco Bonsante. Flat spacetimes with compact hyperbolic cauchy surfaces. J. Differential Geom., 69:441-521, 2005.

[7] M. Cahen and Y. Kerbrat. Transformations conformes des espaces symétriques pseudo-riemanniens. Ann. Mat. Pura Appl. (4), 132:275289 (1983), 1982.

[8] Yvonne Choquet-Brouhat. Théorème d'existence pour certaines systèmes d'équations aux dérivées partielles non linéaires. Acta Math., 88:141-225, 1952.

[9] Yvonne Choquet-Bruhat and Robert Geroch. Global aspects of the cauchy problem in general relativity. Comm. Math. Phys., 14:329335, 1969.

[10] Charles Frances. Géométrie et Dynamique Lorentziennes Conforme. $\mathrm{PhD}$ thesis, Ecole normale supérieure de Lyon, 2002.

[11] Charles Frances. Sur les variétés lorentziennes dont le groupe conforme est essentiel. Math. Ann., 332(1):103-119, 2005.

[12] R. Geroch. Spinor structure of space-times in general relativity ii. J. Mathematical Phys., 83:342-348, 1970.

[13] William M. Goldman. Geometric structures on manifolds and varieties of representations. In Geometry of group representations (Boulder, CO, 1987), volume 74 of Contemp. Math., pages 169-198. Amer. Math. Soc., Providence, RI, 1988.

[14] S. W. Hawking and G. F. R. Ellis. The large scale structure of spacetime. Cambridge University Press, London, 1973. Cambridge Monographs on Mathematical Physics, No. 1.

[15] S. W. Hawking, A. R. King, and P. J. McCarthy. A new topology for curved space-time which incorporates the causal, differential, and conformal structures. J. Mathematical Phys., 17(2):174-181, 1976.

[16] Shoshichi Kobayashi. Transformation groups in differential geometry. Classics in Mathematics. Springer-Verlag, Berlin, 1995. Reprint of the 1972 edition.

[17] Shigenori Matsumoto. Foundations of flat conformal structure. In Aspects of low-dimensional manifolds, volume 20 of Adv. Stud. Pure Math., pages 167-261. Kinokuniya, Tokyo, 1992.

[18] Geoffrey Mess. Lorentz spacetimes of constant curvature. Geom. Dedicata, 126:3-45, 2007.

[19] Ettore Minguzzi and Miguel Sánchez. The causal hierarchy of spacetimes. In Recent developments in pseudo-Riemannian geometry, ESI Lect. Math. Phys., pages 299-358. Eur. Math. Soc., Zürich, 2008.

[20] Barrett O Neil. Semi-Riemannian Geometry. A Series of Monographs and Textbooks. Samuel Eilenberg and Hyman Bass, Orlando, 1983. 
[21] Roger Penrose. Techniques of differential topology in relativity. Society for Industrial and Applied Mathematics, Philadelphia, Pa., 1972. Conference Board of the Mathematical Sciences Regional Conference Series in Applied Mathematics, No. 7.

[22] Roger Penrose. Republication of: Conformal treatment of infinity. Gen. Relativity Gravitation, 43(3):901-922, 2011.

[23] Ernst Alfred Ruh. On automorphism group of a G-structure. PhD thesis, Brown University, 1864.

[24] R. K. Sachs and H. Wu. General relativity and cosmology. Bull. Amer. Math. Soc., 83:1101-1164, 1977. 